\title{
Error Estimates for Binomial Approximations of Game Put Options
}

\author{
Yonatan Iron and Yuri Kifer \\ Institute of Mathematics, Hebrew University, 91904 Jerusalem, Israel \\ Correspondence should be addressed to Yuri Kifer; kifer@math.huji.ac.il
}

Received 17 October 2013; Accepted 21 November 2013; Published 30 January 2014

Academic Editors: P. E. Jorgensen and M. Montero

Copyright (c) 2014 Y. Iron and Y. Kifer. This is an open access article distributed under the Creative Commons Attribution License, which permits unrestricted use, distribution, and reproduction in any medium, provided the original work is properly cited.

\begin{abstract}
A game or Israeli option is an American style option where both the writer and the holder have the right to terminate the contract before the expiration time. Kifer (2000) shows that the fair price for this option can be expressed as the value of a Dynkin game. In general, there are no explicit formulas for fair prices of American and game options and approximations are used for their computations. The paper by Lamberton (1998) provides error estimates for binomial approximation of American put options and here we extend the approach of Lamberton (1998) in order to obtain error estimates for binomial approximations of game put options which is more complicated as it requires us to deal with two free boundaries corresponding to the writer and to the holder of the game option.
\end{abstract}

\section{Introduction}

A put option on a stock can be interpreted as a contract between a holder and a writer which allows the former to claim from the latter at an exercise time $t$ the amount $\left(K-S_{t}\right)^{+}$, where $K$ is a fixed amount called the option's strike, $S_{t}$ is the stock price at time $t$, and $(x)^{+}=\max (x, 0)$. In the American options case its holder has the right to choose any exercise time before the contract matures, while in the game options case the contract writer also has the right to terminate it at any time before its maturity, but then he is required to pay a cancellation fee in addition to the payoff above.

The fair price of American options and of game options is defined as the minimal amount the writer needs to construct a self-financing portfolio which covers his obligation to pay according to the option's contract. It is well known that in the American options case the fair price can be obtained as a value of an appropriate optimal stopping problem, while for game options we have to deal with an optimal stopping (Dynkin) game (see [1]). For more information about results on Dynkin games and game options we refer the reader to the survey [2]. In general, both for American options and, even more so, for game options with finite maturity explicit formulas for their price are not available and approximation methods come into the picture, while estimates of their errors become important. One of the most easily implemented methods is the binomial approximation of stock prices modelled by the geometric Brownian motion and [3] provided corresponding error estimates for American put options. In the present paper we extend this approach in order to provide error estimates of binomial approximations for game put options. We observe that for perpetual game options some explicit formulas can be obtained (see [4]), but the finite maturity case studied here seems to be more realistic.

Approximating the Brownian motion by appropriately normalized sums of Bernoulli random variables the paper [3] provided (error) estimates const $\cdot n^{-3 / 4}$ and const $\cdot n^{-2 / 3}$ for the difference between the price of an American put option and the price of its corresponding $n$th binomial model approximation. Using again the binomial approximation of the Brownian motion as above we construct in this paper two approximating procedures such that the difference between the price of a game put option and its $n$th approximation in the first procedure is between const $\cdot n^{-3 / 4}$ and const $\cdot n^{-1 / 2}$ and in the second procedure is between const. $n^{-1 / 2}$ and const $\cdot n^{-2 / 3}$. The error estimates here are somewhat worse than in the case of American put options which is due to the lack of a smooth fit on the boundary of the writer's stopping 
region which causes substantial difficulties in the study of regularity of payoff functions.

We observe that specific properties of game put options had to be used in order to obtain error estimates with the above precision. For instance, when payoffs are path dependent (and not only dependent on the present value of the stock) [5] provides error estimates of similar binomial approximations only of order $n^{-1 / 4}(\ln n)^{3 / 4}$. Since price functions of game options can be represented as solutions of doubly reflected backward stochastic differential equations the results of [6] are also related to game options approximations. Nevertheless, approximations in [6] are not by binomial models, where computations can be done by means of the effective dynamical programming algorithm (see [5]), but by time discretizations, and so relevant probability space and $\sigma$-algebras remain infinite which prevents effective computations. Furthermore, error estimates in [6] applied to our situation are of order $n^{-1 / 4}$; that is, they are worse than for binomial approximations which we construct here for the specific case of game put options.

Our exposition proceeds as follows. In Section 2 we provide basic results concerning game put option price functions, introduce our approximation processes, and formulate our main result Theorem 1 . In Section 3 we show that the price function can be represented as a solution of a variational inequality problem closely related to the Stefan problem (see [7]). We then use this representation to study regularity properties of the price function near the free boundary of the option's holder exercise region. In Section 4 we study the price function near the boundary of the exercise region of the writer. We use the information about this region from [8] in order to represent the price function as an explicit solution of the heat equation. This representation enables us to understand better the behavior of the price function near the boundary. We estimate also the rate of decay of the price function when the initial stock price tends to infinity. Section 5 is devoted to the proof of Theorem 1. Finally, in Section 6 we exhibit some computations of the price functions and of the free boundaries.

\section{Preliminaries and Main Results}

The Black-Scholes (BS) model of a financial market consists of two assets among which one is nonrisky and the other one is risky. A nonrisky asset is called a bond and its price $B_{t}$ at time $t$ is given by the formula $B_{t}=B_{0} e^{r t}$, where $r$ is interpreted as the interest rate. A risky asset is called a stock and its price at time $t$ is determined by a geometric Brownian motion

$$
S_{t}=S_{0} \exp \left(\left(r-\frac{\kappa^{2}}{2}\right) t+\kappa W_{t}\right)
$$

where $\kappa>0$ is called volatility and $W_{t}, t \geq 0$ is a standard Brownian motion defined on a complete probability space $(\Omega, \mathscr{F}, \mathbf{P})$. If $S_{0}=x$ we write also $S_{t}^{x}$ for $S_{t}$. The fair price of an American put option at time $t$ with a strike (price) $K$ and a maturity (horizon) time $T<\infty$ can now be written as a function $F_{A}\left(t, S_{t}\right)$ of time and the current stock price having the form (see, e.g., [9])

$$
\begin{aligned}
& F_{A}(t, x) \\
& =\sup _{\tau \in \mathscr{T}_{0, T-t}} \operatorname{E} \exp (-r \tau)\left(K-x \exp \left(\left(r-\frac{\kappa^{2}}{2}\right) \tau+\kappa W_{\tau}\right)\right)^{+},
\end{aligned}
$$

where $\mathscr{T}_{0, T-t}$ denotes the set of all stopping times of the Brownian filtration with values in the interval $[0, T-t]$ and $\mathbf{E}$ is the expectation with respect to the measure $\mathbf{P}$. If we set $\psi(x)=\left(K-e^{x}\right)^{+}, P_{A}(t, x)=F_{A}\left(t, e^{x}\right)$, and $\mu=r-\kappa^{2} / 2$ then we can rewrite (2) in the form

$$
P_{A}(t, x)=\sup _{\tau \in \mathscr{T}_{0, T-t}} \mathrm{E} \exp (-r \tau) \psi\left(x+\mu \tau+\kappa W_{\tau}\right) .
$$

Relying on [1] (see also $[4,8,10]$ ) we can also write the fair price of a game put option at time $t$ with a strike price $K$, a maturity time $T$, and a constant penalty $\delta>0$ as a function $F\left(t, S_{t}\right)$ of time and the current stock price in the form

$$
F(t, x)=\inf _{\sigma \in \mathscr{T}_{0, T-t}} \sup _{\tau \in \mathscr{T}_{0, T-t}} \mathbf{E} \exp (-r \sigma \wedge \tau) R(\sigma, \tau),
$$

where $R(s, t)=\left(K-S_{t}^{x}\right)^{+}+\delta \rrbracket_{s<t}$ and $\square_{\mathrm{Q}}$ is the indicator of an event $Q$. Using the functions $P(t, x)=F_{A}\left(t, e^{x}\right)$ and $\psi$ as above we can rewrite this formula in the form

$$
\begin{aligned}
P(t, x)= & \inf _{\sigma \in \mathscr{T}_{0, T-t}} \sup _{\tau \in \mathscr{T}_{0, T-t}} \operatorname{E} \exp (-r \sigma \wedge \tau) \\
& \times\left(\psi\left(x+\mu \sigma \wedge \tau+\kappa W_{\sigma \wedge \tau}\right)+\delta \rrbracket_{\sigma<\tau}\right) .
\end{aligned}
$$

It follows also (see $[1,8,10,11])$ that the saddle point (optimal) stopping times for the game value expressions (4) and (5) are given by

$$
\begin{gathered}
\sigma^{*}=\inf \left\{s<T-t: F\left(t+s, S_{s}^{x}\right)=\left(K-S_{s}^{x}\right)^{+}+\delta\right\} \wedge T, \\
\tau^{*}=\inf \left\{s<T-t: F\left(t+s, S_{s}^{x}\right)=\left(K-S_{s}^{x}\right)^{+}\right\} \wedge T .
\end{gathered}
$$

Next, we introduce our binomial approximations of the Brownian motion

$$
W_{t}^{(n)}=\frac{\sqrt{T}}{\sqrt{n}} \sum_{k=1}^{[n t / T]} \epsilon_{k}, \quad t \in[0, T], n=1,2, \ldots,
$$

where $\epsilon_{k}, k=1,2, \ldots$ are independent identically distributed (i.i.d.) random variables taking on values 1 and -1 with probability $1 / 2$ and $[a]$ denotes the integral part of a number $a$. It is convenient to view $\left\{\epsilon_{k}\right\}_{k=1}^{\infty}$ as defined on the sequence space $\Omega_{\epsilon}=\{-1,1\}^{\mathbb{N}}=\left\{\xi=\left(\xi_{1}, \xi_{2}, \ldots\right): \xi_{i}= \pm 1\right\}$ by the formula $\epsilon_{k}(\xi)=\xi_{k}$ if $\xi=\left(\xi_{1}, \xi_{2}, \ldots\right)$. Then $W_{t}^{(m)}$ will be defined on the probability space $\left(\Omega_{\epsilon}, \mathscr{F}_{\epsilon}, \mathscr{P}_{\epsilon}\right)$, where $\mathscr{P}_{\epsilon}=$ $\{1 / 2,1 / 2\}^{\mathbb{N}}$ is the product measure and $\mathscr{F}_{\epsilon}$ is generated by cylinder sets.

Now set $\delta^{*}=F_{A}(0, K)$ which is the price of the American put option with a maturity $T$ and a strike $K$ provided the 
initial stock price is $K$. It is easy to see that if the penalty $\delta \geq \delta^{*}$ then it does not make sense for the writer to cancel the corresponding game put option (see Lemma 3.1 in [10]), and so in this case the prices of American and game options are the same; that is, $F_{A}(0, K)=F(0, K)$. Since approximations of American options were studied in [3] we assume in this paper that $\delta<\delta^{*}$. Observe that $F_{A}(t, K)$ is continuous in $t$ and it is strictly decreasing to 0 as $t$ increases to $T$, and so for each $\delta \epsilon$ $\left[0, \delta^{*}\right]$ there exists a unique $t_{\delta}<T$ such that $F_{A}\left(t_{\delta}, K\right)=\delta$. Furthermore, we can define $k_{n}$ to be the minimal $k \in \mathbb{N}$ such that $\delta \geq F_{A}(T k / n, K)$ and set $\beta^{(n)}=T k_{n} / n$. In order to define two sequences of functions $P_{1}^{(n)}$ and $P_{2}^{(n)}, n=1,2, \ldots$ which will approximate $P(0, x)$ we set $X_{t}^{(n)}=x+\kappa W_{t}^{(n)}, h=T / n$ and introduce stopping times

$$
\begin{aligned}
\sigma^{(n)}(s)=\inf \{t \in[0, s): & \ln K-|\mu| h-2 \kappa \sqrt{h} \\
& <\mu h\left[\frac{t}{h}\right]+X_{t}^{(n)} \\
& <\ln K+|\mu| h+2 \kappa \sqrt{h}\} \wedge T,
\end{aligned}
$$

where $\sigma^{(n)}=T$ if the infimum above is taken over the empty set and we set $\sigma^{(n)}=\sigma^{(n)}\left(\beta^{(n)}\right)$. Introduce a filtration $\left\{\mathscr{G}_{t}=\right.$ $\left.\mathscr{F}_{[t / h]}, t \geq 0\right\}$, where $\mathscr{F}_{0}$ is the trivial $\sigma$-algebra and $\mathscr{F}_{k}$ is generated by $\epsilon_{1}, \ldots, \epsilon_{k}$. Denote by $\mathscr{T}^{(n)}$ the set of all stopping times with respect to the filtration $\left\{\mathscr{G}_{t}\right\}$ taking on value in the set $\{k h, k=0,1, \ldots, n\}$. Then, clearly, $\sigma^{(n)} \in \mathscr{T}^{(n)}$. Now, for $x \leq \ln K$ we define

$$
\begin{aligned}
P_{1}^{(n)}(s, x)=\sup _{\tau \in \mathscr{T}^{(n)}} \mathrm{E}\left(e^{-r \sigma^{(n)}(s) \wedge \tau}(\right. & \psi\left(\mu \tau+X_{\tau}^{(n)}\right) \rrbracket_{\left\{\tau \leq \sigma^{(n)}(s)\right\}} \\
& \left.\left.+\delta \rrbracket_{\left\{\sigma^{(n)}(s)<\tau\right\}}\right)\right),
\end{aligned}
$$

and for $x>\ln K$ we set

$$
\begin{aligned}
& P_{1}^{(n)}(s, x) \\
& =\sup _{\tau \in \mathscr{T}^{(n)}} \mathbf{E}\left(e ^ { - r \sigma ^ { ( n ) } ( s ) \wedge \tau } \left(\psi\left(\mu \tau+X_{\tau}^{(n)}\right) \mathbb{q}_{\left\{\tau \leq \sigma^{(n)}(s)\right\}}\right.\right. \\
& +\left(\delta-K e^{(|\mu| \sqrt{h}+\kappa h)}\right) \\
& \left.\left.\times \rrbracket_{\left\{\sigma^{(n)}(s)<\tau\right\}}\right)\right) .
\end{aligned}
$$

The second approximation function is defined for all $x$ by

$$
\begin{aligned}
P_{2}^{(n)}(s, x) & \\
=\sup _{\tau \in \mathscr{T}^{(n)}} \mathrm{E}\left(e^{-r \sigma^{(n)}(s) \wedge \tau}(\right. & \psi\left(\mu \tau+X_{\tau}^{(n)}\right) \mathbb{q}_{\left\{\tau \leq \sigma^{(n)}(s)\right\}} \\
& +\left(\psi\left(\mu \sigma^{(n)}(s)+X_{\sigma^{(n)}(s)}^{(n)}\right)+\delta\right) \\
& \left.\left.\times \mathbb{q}_{\left\{\sigma^{(n)}<\tau\right\}}\right)\right) .
\end{aligned}
$$

Setting $P_{i}^{(n)}(x)=P_{i}^{(n)}\left(\beta^{(n)}, x\right)$ we formulate now our main result.
Theorem 1. For each $x$ there exists $C=C(x)$ such that for all $n=1,2, \ldots$,

$$
\begin{aligned}
& -\frac{C}{n^{1 / 2}} \leq P_{1}^{(n)}(x)-P(0, x) \leq \frac{C}{n^{3 / 4}}, \\
& -\frac{C}{n^{2 / 3}} \leq P_{2}^{(n)}(x)-P(0, x) \leq \frac{C}{n^{1 / 2}} .
\end{aligned}
$$

Observe that $P_{i}^{(n)}(x), i=1,2$ appearing in Theorem 1 is defined via $\beta^{(n)}$ and $k_{n}$ which can be obtained only knowing precise price function $F_{A}(t, K)$ of the American put option with the initial stock price equal to $K$. But from the computational point of view we can obtain $F_{A}$ only approximately using, for instance, the algorithm from [3]. One of the ways to overcome this difficulty is to proceed as follows. Let $F_{A}^{(n)}(t, K)$ denote the $n$th binomial approximation of $F_{A}^{(n)}(t, K)$ obtained in [3] which uniformly in $t \in[0, T]$ satisfies

$$
-\frac{\tilde{c}}{n^{2 / 3}} \leq F_{A}^{(n)}(t, K)-F_{A}(t, K) \leq \frac{\widetilde{C}}{n^{3 / 4}}
$$

for some $\widetilde{c}, \widetilde{C}>0$. Denote by $m_{n}$ the minimal $m \leq n$ such that $\delta \geq F_{A}^{(n)}(T m / n, K)$ taking $m_{n}=n$ if this inequality does not hold true for all $m \leq n$. Set $\gamma^{(n)}=T m_{n} / n$ which unlike $\beta^{(n)}$ can be computed employing [3]. It is well known that $\partial F_{A}(t, K) / \partial t$ exists (see, e.g., [3]) and, clearly, this derivative is nonpositive. In fact, it is possible to show that

$$
\frac{\partial F_{A}(t, K)}{\partial t}<0, \quad d=\inf _{0<t<T}\left|\frac{\partial F_{A}(t, K)}{\partial t}\right|>0 .
$$

This together with (13) yields that

$$
-\frac{\widetilde{C}}{d n^{3 / 4}} \leq \beta^{(n)}-\gamma^{(n)} \leq \frac{\widetilde{c}}{d n^{2 / 3}} .
$$

From the definitions (9)-(11) it follows that for each $x>0$ there exists $\widetilde{\widetilde{C}}=\widetilde{\widetilde{C}}(x)>0$ independent of $s, \widetilde{s} \in[0, T]$ such that

$$
\left|P_{i}^{(n)}(s, x)-P_{i}^{(n)}(\widetilde{s}, x)\right| \leq \widetilde{\widetilde{C}}|s-\widetilde{s}|, \quad i=1,2 .
$$

Now we obtain from Theorem 1 together with (15) and (16) the following.

Corollary 2. For each $x>0$ there exists $C=C(x)>0$ such that for all $n=1,2, \ldots$,

$$
\begin{aligned}
-\frac{C}{n^{1 / 2}} & \leq P_{1}^{(n)}\left(\gamma^{(n)}, x\right)-P(0, x) \leq \frac{C}{n^{2 / 3}}, \\
-\frac{C}{n^{2 / 3}} & \leq P_{1}^{(n)}\left(\gamma^{(n)}, x\right)-P(0, x) \leq \frac{C}{n^{1 / 2}} .
\end{aligned}
$$

In the following sections we will analyze regularity properties of the price function $P(t, x)$ of game put options and will complete the proof of Theorem 1 in Section 5 providing some computations in Section 6. The general strategy of the proof resembles that of [3], but the study of the price function 
of game put options is more complicated than in the American options case, in particular, because of appearance of two exercise boundaries (holder's and writer's) having different properties. Our proof will be based on regularity properties of solutions of parabolic partial differential equations with free boundary and of the corresponding variational inequalities and we will rely also on some prior results from $[3,4,8]$.

\section{Price Function Near the Holder's Exercise Boundary}

3.1. Some Previous Results. First, we state the following result from [8] (see also [10]) which we will use later on.

Proposition 3. (i) There exists an increasing function $b$ : $[0, T) \rightarrow \mathbb{R}$ such that $\lim _{t \rightarrow T} b(t)=K$ and $F(t, x)=K-x$ for all $(t, x)$ satisfying $0<x \leq b(t)$.

(ii) There exists $0<\delta^{*}$ such that for every $0 \leq \delta \leq \delta^{*}$ there is a $\beta=\beta(\delta) \geq 0$ so that $F(t, K)=\delta$ for $t \in[0, \beta]$ and for $t \geq \beta$ one has $F(t, x)=F_{A}(t, x)$ for all $x \geq 0$.

(iii) Furthermore,

$$
F_{t}(t, x)+\frac{1}{2} \kappa^{2} x^{2} F_{x x}(t, x)+r x F_{x}(t, x)-r F(t, x)=0,
$$

for all

$$
(t, x) \in(0, T) \times \mathbb{R}^{+} \backslash(\{(t, x): x \leq b(t)\} \cup[0, \beta) \times\{K\}) .
$$

In particular, $F(t, x)$ is of class $C^{1,2}$, that is, continuously differentiable once with respect to $t$ and twice with respect to $x$, and so, in fact, it is a smooth function there.

(iv) Finally, $F(t, x)$ is convex and strictly decreasing in $x$ and nonincreasing in $t$.

Next, we introduce an operator $\mathscr{D}$ which acts on Borel functions $u(t, x)$ on $[0, T] \times \mathbb{R}$ by

$$
\begin{aligned}
& \mathscr{D u}(t, x) \\
& \quad=\frac{1}{2}[u(t+h, x+\kappa \sqrt{h})+u(t+h, x-\kappa \sqrt{h})]-u(t, x) .
\end{aligned}
$$

Clearly, $(1 / h) \mathscr{D}(t, x)$ can be viewed as a discretization of the differential operator $(\partial / \partial t)+\left(\kappa^{2} / 2\right)\left(\partial^{2} / \partial x^{2}\right)$. We will rely on the following results from [3] concerning the operator $\mathscr{D}$.

Proposition 4. For each Borel function $u$ on $[0, T] \times \mathbb{R}$ there exists a martingale $\left(M_{t}\right)_{0 \leq t \leq T}$ with respect to the filtration $\mathscr{G}_{t}, t \geq 0$ such that $M_{0}=0$ and for every $t \in\{0, h, 2 h, \ldots, T\}$,

$$
u\left(t, X_{t}^{(n)}\right)=u(0, x)+M_{t}+\sum_{j=1}^{n t / T} \mathscr{D} u\left((j-1) h, X_{(j-1) h}^{(n)}\right)
$$

Proposition 5. Let $0 \leq t \leq T-h$ and $x \in \mathbb{R}$. Assume that $u$ is a $C^{2}$ function on $([t, t+h] \times[x+\kappa \sqrt{h}, x-\kappa \sqrt{h}])$. Then

$$
\begin{array}{r}
\mathscr{D u}(t, x) \\
=\frac{1}{\kappa} \int_{0}^{\sqrt{h}} d y \int_{-\kappa y}^{\kappa y} d z\left(z \frac{\partial^{2} u}{\partial t \partial x}\left(t+y^{2}, x+z\right)\right. \\
\left.+\delta(u)\left(t+y^{2}, x+z\right)\right),
\end{array}
$$

where

$$
\delta(u)(t, x)=u_{t}(t, x)+\frac{\kappa^{2}}{2} u_{x x}(t, x) .
$$

We will need also the following result concerning the free boundary $s(t)=\ln (b(t))$ of the holder exercise region of our game put option which in the case of American options appears as Proposition 1 in [3] and it can be proved for game options in the same way.

Proposition 6. Let $0 \leq t_{1}<t_{2} \leq \beta$ and let $x_{0}=s(0)<$ $x_{1}=s(\beta)<\ln K$; then $\left(s\left(t_{1}\right)-s\left(t_{2}\right)\right)^{2} \leq \sup _{x_{0} \leq x \leq x_{1}} \mid P\left(t_{1}, x\right)-$ $P\left(t_{2}, x\right) \mid$.

We also observe that it follows from the Berry-Esseen estimate (see [12]) that, for some constant $C_{1}>0$ independent of $j, n \geq 1$, and $z \in \mathbb{R}$,

$$
\mathbf{P}\left\{\left|X_{j h}^{(n)}-z\right| \leq \kappa \sqrt{h}\right\} \leq \frac{C_{1}}{\sqrt{j}} .
$$

We will also rely on the following standard bounds on derivatives of solutions of 2 nd-order parabolic equations with constant coefficients (see, e.g., $[13,14]$ ).

Proposition 7. Let $D=(0, T) \times(0,1)$ and let $w(t, x) \in C[\bar{D}]$ be a solution in $D$ of the following parabolic equation:

$$
\frac{\kappa^{2}}{2} w_{x x}+\mu w_{x}-r w=w_{t}
$$

Suppose that $w(0, x)=0$ for all $0 \leq x \leq 1$ and that there exists $A>0$ such that $|w(t, x)|<A$ for all $(x, t) \in \bar{D}$. Then for every $k, n$ and $0<a<b<1$ there exists $C=C(k, n, a, b, T, A)$ such that

$$
\left|\frac{\partial^{k+n} w}{\partial^{k} x \partial^{n} t}(t, x)\right|<C \quad \forall(t, x) \in(0, T) \times[a, b] .
$$

3.2. Price Function and Variational Inequalities. Next, we will show that the price function of the game put option can be represented as a solution of a variational inequality (v.i.) problem which is a generalization of the Stefan problem (see [7], VIII). This will enable us to derive certain regularity properties of this price function which we will use later on. Details of some of the proofs concerning the solutions of the v.i. problem below which are similar to the proofs in the case of the Stefan problem will not be given here. For the 
corresponding results in the American put option case we refer the reader to $[3,9]$ and to references there in.

Let $T^{\prime}$ be such that $\beta<T^{\prime}<T$ and set

$$
\mathbf{A}=\frac{\kappa^{2}}{2} \frac{\partial^{2}}{\partial x^{2}}+\mu \frac{\partial}{\partial x}-r, \quad \text { where } \mu=r-\frac{\kappa^{2}}{2} .
$$

Using the maximum principle, properties of price functions of American and game put options, and the fact that after time $\beta$ the price functions of the game and American option are the same we obtain that for every $x>s\left(T^{\prime}\right)$ the time derivative $P_{t}\left(T^{\prime}, x\right)=P_{A, t}\left(T^{\prime}, x\right)$ is strictly negative and we can find $a, b$ satisfying $s\left(T^{\prime}\right)<a<b<\ln K$ such that, for some constant $c>0$,

$$
-P_{t}\left(T^{\prime}, x\right)>c \quad \forall x \in[a, b] .
$$

Relying on Proposition 3(iii) we also observe that for all $(t, x) \in\left[0, T^{\prime}\right] \times(s(t), \ln K)$,

$$
\begin{gathered}
\frac{\partial P}{\partial t}(t, x)+\mathbf{A} P(t, x)=0, \\
P(t, x)>K-e^{x} \quad \forall(t, x) \in\left[0, T^{\prime}\right] \times(s(t), \ln K), \\
P(t, x)=K-e^{x} \quad \forall t \in\left[0, T^{\prime}\right], \forall x \leq s(t), P_{t} \leq 0 .
\end{gathered}
$$

Let $a_{0}$ be such that $a_{0}<s(0)<s\left(T^{\prime}\right)<b$. Introduce the domain $D=\left(0, T^{\prime}\right) \times\left(a_{0}, b\right)$ and for all $(t, x)$ in the closure $\bar{D}$ of $D$ define the functions

$$
v(t, x)=P\left(T^{\prime}-t, x\right)-P\left(T^{\prime}, x\right), \quad f(x)=\mathbf{A} P\left(T^{\prime}, x\right) .
$$

We obtain that

$$
f(x)= \begin{cases}-P_{t}\left(T^{\prime}, x\right), & s\left(T^{\prime}\right)<x \leq b \\ -r K, & a_{0} \leq x \leq s\left(T^{\prime}\right)\end{cases}
$$

and from the definition of $v(t, x)$ it follows that for any $(t, x) \epsilon$ $\bar{D}$

$$
\begin{gathered}
P_{t}\left(T^{\prime}-t, x\right)=-v_{t}(t, x), \\
P_{t x}\left(T^{\prime}-t, x\right)=-v_{t x}(t, x), \\
P_{t t}\left(T^{\prime}-t, x\right)=v_{t t}(t, x), \\
P_{x}\left(T^{\prime}-t, x\right)-P_{x}\left(T^{\prime}, x\right)=v_{x}(t, x), \\
P_{x x}\left(T^{\prime}-t, x\right)-P_{x x}\left(T^{\prime}, x\right)=v_{x x}(t, x) .
\end{gathered}
$$

Since $P_{x}\left(T^{\prime}, x\right)$ and $P_{x x}\left(T^{\prime}, x\right)$ are bounded we obtain that the integrability properties of the first- and second-order derivatives of $P(t, x)$ and $v(t, x)$ are the same in $\bar{D}$. Now set

$$
\psi(t)=v(t, b), \quad g(t)=v_{t}(t, b) \quad \text { for } 0 \leq t \leq T^{\prime} .
$$

Then by (28) and (32),

$$
\psi(t)=\int_{0}^{t} g(\tau) d \tau=\int_{0}^{t} v_{t}(\tau, b) d \tau \geq 0 \quad \text { for } 0 \leq t \leq T^{\prime} .
$$

It follows from (29) and (30)-(31) that on the set $v>0$,

$$
\begin{aligned}
& v_{t}-\mathbf{A} v-f \\
& =-P_{t}\left(T^{\prime}-t, x\right)-\mathbf{A} P\left(T^{\prime}-t, x\right)+\mathbf{A} P\left(T^{\prime}, x\right)-f(x)=0,
\end{aligned}
$$

and on the set $v=0$ we obtain

$$
v_{t}-\mathbf{A} v-f=r K>0 .
$$

Hence we arrive at the following (see [7]).

Lemma 8. The function $v$ is the unique solution of the following variational inequality problem.

v.i. Problem 1. Find $v \in L^{2}\left[0, T^{\prime} ; H^{2}\left(a_{0}, b\right)\right] \cap H^{1}[D]$ such that

(i) $v, v_{t} \geq 0$,

(ii) $\left(v_{t}-\mathbf{A} v\right)(w-v) \geq f(w-v)$ a.s for every $w \in$ $L^{2}[D], w \geq 0$

(iii) $v(t, b)=\psi$ for $0 \leq t \leq T^{\prime}, x=b$,

(iv) $v\left(t, a_{0}\right)=0$ for $0 \leq t \leq T^{\prime}, x=a_{0}$,

(v) $v(0, x)=0$ for $t=0, a_{0} \leq x \leq b$.

Proof. We will prove uniqueness and the fact that $v$ is a solution to v.i. Problem 1 follows from (30)-(36). Assume that $v$ and $\widetilde{v}$ are two solutions of v.i. Problem 1 . Since $\widetilde{v} \geq 0$ (property (i)) we can use the property (ii) of $v$ and replace $w$ by $\widetilde{v}$. Since both of them are solutions we obtain that

$$
\begin{aligned}
& \left(v_{t}-\mathbf{A} v\right)(\widetilde{v}-v) \geq f(\widetilde{v}-v), \\
& \left(\widetilde{v}_{t}-\mathbf{A} \widetilde{v}\right)(v-\widetilde{v}) \geq f(v-\widetilde{v}) .
\end{aligned}
$$

Define the parabolic boundary as the boundary of $D$ without the interval $\left\{T^{\prime}\right\} \times\left(a_{0}, b\right)$ and let $u=v-\widetilde{v}$. Note that $u$ is zero on the parabolic boundary and the sum of the two inequalities (37) is

$$
u_{t} u-\frac{\kappa^{2}}{2} u_{x x} u-\mu u_{x} u+r u^{2}=\left(u_{t}-\mathbf{A} u\right) u \leq 0 .
$$

Integrating both sides of $(38)$ on $\left(0, T^{\prime}\right) \times\left(a_{0}, b\right)$ we obtain four terms on the left side. For the first term we have

$$
\int_{a_{0}}^{b} \int_{0}^{T^{\prime}} u(t, x) u_{t}(t, x) d t d x=\int_{a_{0}}^{b} \frac{1}{2} u^{2}\left(T^{\prime}, x\right) d x \geq 0
$$

Integration by parts of the second term and the fact that $u=0$ on the parabolic boundary yield

$$
\begin{array}{r}
-\frac{\kappa^{2}}{2} \int_{0}^{T^{\prime}} \int_{a_{0}}^{b} u_{x x}(t, x) u(t, x) d x d t \\
\quad=\frac{\kappa^{2}}{2} \int_{0}^{T^{\prime}} \int_{a_{0}}^{b} u_{x}^{2}(t, x) d x d t \geq 0 .
\end{array}
$$

For the third term note that $u_{x} u=(1 / 2)\left(d u^{2} / d x\right)$ and that $u\left(t, a_{0}\right)=u\left(t, b_{0}\right)=0$ for every $t$, and so

$$
\mu \int_{0}^{T^{\prime}} \int_{a_{0}}^{b} u_{x}(t, x) u(t, x) d x d t=0
$$


The last term satisfies $r \int_{0}^{T^{\prime}} \int_{a_{0}}^{b} u^{2}(t, x) d x d t \geq 0$ since $r>0$. We conclude that the left side of (38) cannot be negative and so it must be zero. Since all terms in the left-hand side of (38) are nonnegative and their sum is equal to 0 we obtain that $r \int_{0}^{T^{\prime}} \int_{a_{0}}^{b} u^{2}(t, x) d x d t=0$, and so $u=0$ almost everywhere (a.e.). Hence, $v=\widetilde{v}$ a.e., and so there is only one continuous solution.

Denote parts of the boundary of $D=\left(0, T^{\prime}\right) \times\left(a_{0}, b\right)$ by

$$
\begin{array}{ll}
\Gamma_{1}=\left[0, T^{\prime}\right] \times\{b\}, & \Gamma_{2}=\{0\} \times\left(a_{0}, b\right), \\
\Gamma_{3}=\left[0, T^{\prime}\right] \times\left\{a_{0}\right\}, & \Gamma=\Gamma_{1} \cup \Gamma_{2} \cup \Gamma_{3}
\end{array}
$$

and set

$$
\mathbf{L}=\frac{\partial}{\partial t}-\mathbf{A}=\frac{\partial}{\partial t}-\frac{\kappa^{2}}{2} \frac{\partial^{2}}{\partial x^{2}}-\mu \frac{\partial}{\partial x}+r .
$$

Thus, $\Gamma$ is a parabolic boundary of $D$. For every $\varepsilon>0$ we define following functions.

(1) A smooth function $f^{(\varepsilon)}(x) \geq f(x)$ on $\left(a_{0}, b\right)$ such that $f^{(\varepsilon)}(x)=f(x)$ for $s\left(T^{\prime}\right)<a<x \leq b$ and for $a_{0} \leq x \leq a_{1}$, where $a_{1}$ satisfies $a_{0}<a_{1}<s\left(T^{\prime}\right)$ and $\lim _{\varepsilon \rightarrow 0} f^{(\varepsilon)}(x)=f(x)$ for $a_{0} \leq x \leq b$.

(2) A smooth function $\beta^{(\varepsilon)}(v)$ satisfying

$$
\begin{gathered}
\beta^{(\varepsilon)}(v)=0 \quad \forall v \geq \varepsilon, \quad \beta^{(\varepsilon)}(0)=-1, \\
\beta_{v}^{(\varepsilon)}(v) \geq 0, \quad \beta_{v v}^{(\varepsilon)}(v) \leq 0 .
\end{gathered}
$$

(3) $\psi^{(\varepsilon)}(t)=\psi(t)+\varepsilon$ with $\psi$ defined in (33).

(4) A smooth function $\eta(x)$ such that $0 \leq \eta(x) \leq 1$ and for some $a<a_{2}<b$,

$$
\begin{array}{ll}
\eta(x)=1 & \text { for } a_{2} \leq x \leq b, \\
\eta(x)=0 & \text { for } a_{0} \leq x \leq a .
\end{array}
$$

Set $F_{\varepsilon}(x, v)=f^{(\varepsilon)}(x)-r K \beta^{(\varepsilon)}(v)$ which is a Lipschitz continuous function and for every constant $C$ there is $M_{0}$ such that $C\left|F^{(\varepsilon)}(x, v)\right| \leq M$ whenever $M \geq M_{0}$ and $|v| \leq M$. Let $\phi^{(\varepsilon)}$ be a function on $\Gamma$ satisfying

$$
\left.\phi^{(\varepsilon)}\right|_{\Gamma_{1}}=\psi^{(\varepsilon)}(t),\left.\quad \phi^{(\varepsilon)}\right|_{\Gamma_{2}}=\varepsilon \eta(x),\left.\quad \phi^{(\varepsilon)}\right|_{\Gamma_{3}}=0,
$$

and, moreover, relying on Chapter 3 in [14] we can choose $\phi^{(\varepsilon)}$ so that

(1) $\phi^{(\varepsilon)} \in \bar{C}_{2+\delta}[D]$ for some $0<\delta<1$ (in fact for each $\delta$ ) and we refer the reader to Chapter 3 in [14] for the definition of $\bar{C}_{2+\delta}[D]$ and for conditions yielding that a function defined only on the boundary $\Gamma$ can be extended to a function from $\bar{C}_{2+\delta}[D]$;

(2) $\mathbf{L} \phi^{(\varepsilon)}=F^{(\varepsilon)}(x, \psi)$ at the points $(0, b)$ and $\left(0, a_{0}\right)$.
By the theory of semilinear parabolic equations (see [14]) there exists a function $v^{(\varepsilon)} \in \bar{C}_{2+\gamma}[D]$ for some $0<\gamma<1$ such that

$$
\mathbf{L} v^{(\varepsilon)}=F^{(\varepsilon)}\left(x, v^{(\varepsilon)}\right),\left.\quad v^{(\varepsilon)}\right|_{\Gamma}=\phi^{(\varepsilon)} .
$$

In particular $v^{(\varepsilon)}, v_{x}^{(\varepsilon)}, v_{x x}^{(\varepsilon)}, v_{t}^{(\varepsilon)}$ are continuous on $\bar{D}$.

Let $w=v_{t}^{(\varepsilon)}$. By differentiating with respect to $t(47)$ and taking into account (33), (47), and the properties of $\phi^{(\varepsilon)}$ we obtain that

$$
\begin{aligned}
& w_{t}-\frac{\kappa^{2}}{2} w_{x x}-\mu w_{x}+\left(r+r K \beta_{v}^{(\varepsilon)}\left(v^{(\varepsilon)}\right)\right) w=0 \\
& \text { where } w(t, b)=g(t) \quad \forall 0 \leq t \leq T^{\prime}, \\
& w\left(t, a_{0}\right)=0 \quad \forall 0 \leq t \leq T^{\prime} \\
& w(0, x)=f^{(\varepsilon)}(x) \\
& +\varepsilon\left(\frac{\kappa^{2}}{2} \eta_{x, x}(x)+\mu \eta_{x}(x)-r \eta(x)\right) \\
& -r K \beta_{v}^{(\varepsilon)}\left(v^{(\varepsilon)}(0, x)\right) \quad \forall a_{0} \leq x \leq b .
\end{aligned}
$$

We see that in $D$ the function $w$ is a solution to a parabolic equation and since $r+r K \beta_{v}^{(\varepsilon)} \geq 0$ we can use the maximum principle

$$
\begin{aligned}
\min \left(\min _{\Gamma}(w), 0\right) & \leq w(t, x) \\
& \leq \max \left(\max _{\Gamma}(w), 0\right) \quad \forall(t, x) \in D .
\end{aligned}
$$

Therefore in order to bound the function $w$ we only need to bound its values on the parabolic boundary. First, we estimate the left hand side of (49). For $a \leq x \leq b$ we have that $v^{(\varepsilon)}(0, x)=\varepsilon \eta(x) \leq \varepsilon$, and so $\beta^{(\varepsilon)}\left(v^{(\varepsilon)}\right) \leq 0$. In view of $(28)$, (31), and the definition of $f^{(\varepsilon)}$ above there exists $\varepsilon_{0}>0$ such that, for every $0<\varepsilon \leq \varepsilon_{0}$,

$$
\begin{aligned}
w(0, x) & \geq f(x)+\varepsilon\left(\eta_{x x}(x)+\mu \eta_{x}(x)-r \eta(x)\right) \\
& =-P_{t}\left(T^{\prime}, x\right)+\varepsilon\left(\frac{\kappa^{2}}{2} \eta_{x x}(x)+\mu \eta_{x}(x)-r \eta(x)\right) \\
& \geq 0 \quad \forall a \leq x \leq b .
\end{aligned}
$$

On the interval $a_{0} \leq x \leq a$ we have $\eta=0$ and since $v^{(\varepsilon)}(0, x)=\varepsilon \eta(x)=0$ we see that $\beta^{(\varepsilon)}\left(v^{(\varepsilon)}(0, x)\right)=-1$. Since on this interval $f(x) \geq-r K$ we obtain

$$
\begin{aligned}
w(0, x) & =f^{(\varepsilon)}(x)-r K \beta^{(\varepsilon)}(0) \\
& \geq f(x)+r K \geq 0 \text { for } a_{0} \leq x<a .
\end{aligned}
$$


Hence, $w \geq 0$ on $\Gamma_{2}$. We obtain next that

$$
\begin{gathered}
w(t, b)=-\frac{\partial P}{\partial t}\left(T^{\prime}-t, b\right) \geq 0 \text { on } \Gamma_{1}, \\
w(t, b)=0 \quad \text { on } \Gamma_{3} .
\end{gathered}
$$

It follows that $\min \left(\min _{\Gamma}(w), 0\right)=0$.

Next, we estimate the right-hand side of (49). On $\Gamma_{2}$ we have that

$$
\begin{aligned}
& w(0, x) \\
& \leq \mid f^{(\varepsilon)}(x)+\varepsilon\left(\frac{\kappa^{2}}{2} \eta_{x x}(x)+\mu \eta_{x}(x)-r \eta(x)\right) \\
& -r K \beta^{(\varepsilon)}\left(v^{(\varepsilon)}(0, x)\right) \\
& \leq \sup \left|f^{(\varepsilon)}\right|+\varepsilon \sup \left|\frac{\kappa^{2}}{2} \eta_{x x}+\mu \eta_{x}-r \eta\right|+r K \leq C_{0} \text {, }
\end{aligned}
$$

where $C_{0}>0$ is a constant independent of $\varepsilon$, and so

$$
\begin{aligned}
0 & =\min \left(\min _{\Gamma}(w), 0\right) \leq w(t, x) \\
& \leq \max \left(\max \left(-\frac{\partial P}{\partial t}\left(T^{\prime}-t, b\right), C_{0}\right)\right)=C_{1} .
\end{aligned}
$$

We conclude that there are some constants $\varepsilon_{0}$ and $C_{1}$ such that for every $0<\varepsilon \leq \varepsilon_{0}$,

$$
0 \leq v_{t}^{(\varepsilon)}(t, x) \leq C_{1} .
$$

Since $v_{t}^{(\varepsilon)} \geq 0$ and $v^{(\varepsilon)}(0, x) \geq 0$ for $a_{0} \leq x \leq b$ we deduce that $v^{(\varepsilon)}(t, x) \geq 0$ and because $v_{t}^{(\varepsilon)}$ is uniformly bounded it follows that $v^{(\varepsilon)}$ is also uniformly bounded. By the properties of $\beta^{(\varepsilon)}$ we see that

$$
-1 \leq \beta^{(\varepsilon)}\left(v^{(\varepsilon)}\right) \leq 0 \quad \text { or } \quad\left\|\beta^{(\varepsilon)}\left(v^{(\varepsilon)}\right)\right\|_{L^{\infty}[D]} \leq 1 .
$$

Let $D_{0}=(0, T) \times\left(a_{2}, b\right)$ be an upper subrectangle of $D$, where $a_{2}$ is the same as in the definition of the function $\eta$ in (4). From the definition we have $v^{(\varepsilon)}(0, x)=\varepsilon \eta(x)=\varepsilon$ in $\bar{D}_{0}$ and since $v_{t}^{(\varepsilon)}$ is nonnegative, we obtain that $v^{(\varepsilon)}(t, x) \geq \varepsilon$, and so $\beta^{(\varepsilon)}\left(v^{(\varepsilon)}(t, x)\right)=0$.

This means that on $D_{0}$ the function $v^{(\varepsilon)}$ satisfies the parabolic equation

$$
\mathbf{L} v^{(\varepsilon)}=f^{(\varepsilon)}
$$

For $w=v_{t}^{(\varepsilon)}$ and $0<\varepsilon<\varepsilon_{0}$ we also have that

$$
\begin{gathered}
\mathbf{L} w(t, x)=0 \quad \forall(t, x) \in D_{0}, \\
w(0, x)=f^{(\varepsilon)}(x) \quad \text { when } a_{2} \leq x \leq b .
\end{gathered}
$$

Next, let $y(t, x)$ be a function on $D_{0}$ such that

$$
\begin{aligned}
& \mathbf{L} y(t, x)=0 \quad \forall(t, x) \in D_{0}, \\
& y(0, x)=f^{(\varepsilon)}(x) \quad \forall a_{2} \leq x \leq b,
\end{aligned}
$$

and all of its first- and second-order derivatives are bounded there. Such a function exists since we can choose a smooth function on the remaining part $\Gamma_{0} \backslash\{0\} \times\left[a_{2}, b\right]$ of the parabolic boundary $\Gamma_{0}$ of $D_{0}$ which extends $f^{(\varepsilon)}(x)$ as a smooth function to the whole $D_{0}$ and then use Theorem 12 from Chapter 3 in [7]. For each $\varepsilon<\varepsilon_{0}$ we define $z(t, x)=$ $w(t, x)-y(t, x)$ in the domain $D_{0}$, where $w(t, x)=v_{t}^{(\varepsilon)}(t, x)$. Then $z(0, x)=w(0, x)-y(0, x)=0$ for every $a_{2} \leq x \leq b$. Fix $x_{0} \in\left(a_{2}, b\right)$; then by Proposition 4.5 from Section 4.1 of [7] we obtain that $\left|z_{t}\left(t, x_{0}\right)\right|,\left|z_{t t}\left(t, x_{0}\right)\right|<C$ for every $0 \leq t \leq T^{\prime}$, where a constant $C>0$ is independent of $\varepsilon$. Since we assume that $\left|y_{t}\left(t, x_{0}\right)\right|,\left|y_{t t}\left(t, x_{0}\right)\right|<C_{1}$ for $0 \leq t \leq T^{\prime}$ it follows that $\left|w_{t}\left(t, x_{0}\right)\right|,\left|w_{t t}\left(t, x_{0}\right)\right|<C_{1}+C$ (for every $\varepsilon$ ). Let $D_{1}=\left(x_{0}, b\right) \times(0, T)$; then by Theorem 6 in Chapter 3 of [14] we obtain that for every $\varepsilon>0$ (and in fact every $0<\alpha<1$ ) $v^{(\varepsilon)}, v_{t}^{(\varepsilon)} \in \bar{C}_{2+\alpha}\left[D_{1}\right]$ and there is a constant $C$ independent of $\varepsilon$ such that

$$
\left.\overline{\mid v^{(\varepsilon)}}\right|_{2+\alpha}+\overline{\left|v_{t}^{(\varepsilon)}\right|_{2+\alpha}}<C .
$$

For the definition of norms in (60) see Chapter 3 in [14].

In particular, we get

$$
\left\|v_{x}^{(\varepsilon)}\right\|_{L^{\infty}\left(D_{1}\right)}+\left\|v_{t x}^{(\varepsilon)}\right\|_{L^{\infty}\left(D_{1}\right)}+\left\|v_{t t}^{(\varepsilon)}\right\|_{L^{\infty}\left(D_{1}\right)}<C .
$$

Considering again the whole region $D$ we have

$$
\begin{gathered}
-\int_{x}^{b} v_{x x}^{(\varepsilon)}(t, y) d y \\
=\frac{2}{\kappa^{2}} \int_{x}^{b}\left(\mu v_{x}^{(\varepsilon)}(t, y)-v_{t}^{(\varepsilon)}(t, y)-r v^{(\varepsilon)}(t, y)\right. \\
\left.\quad+\beta^{(\varepsilon)}\left(v^{(\varepsilon)}(t, y)\right)+f^{(\varepsilon)}(y)\right) d y .
\end{gathered}
$$

Hence,

$$
\begin{aligned}
& v_{x}^{(\varepsilon)}(t, x)=v_{x}^{(\varepsilon)}(t, b) \\
& +\frac{2}{\kappa^{2}}\left[\mu\left(v^{(\varepsilon)}(t, b)-v^{(\varepsilon)}(t, x)\right)\right. \\
& +\int_{x}^{b}\left(-v_{t}^{(\varepsilon)}(t, y)-r v^{(\varepsilon)}(t, y)\right. \\
& \left.\left.\quad+\beta^{(\varepsilon)}\left(v^{(\varepsilon)}(t, y)\right)+f^{(\varepsilon)}(y)\right) d y\right] .
\end{aligned}
$$

Since all terms in the right-hand side are uniformly bounded there is a constant $C>0$ independent of $\varepsilon$ such that $\left\|v_{x}^{(\varepsilon)}\right\|<C$ for every $0<\varepsilon \leq \varepsilon_{0}$. Now we see that in the equation

$$
\begin{aligned}
& v_{x x}^{(\varepsilon)}(t, y)=\frac{2}{\kappa^{2}}\left(\mu v_{x}^{(\varepsilon)}(t, y)-v_{t}^{(\varepsilon)}(t, y)-r v^{(\varepsilon)}(t, y)\right. \\
&\left.+\beta^{(\varepsilon)}\left(v^{(\varepsilon)}(t, y)\right)+f^{(\varepsilon)}(y)\right)
\end{aligned}
$$

all terms in the right hand side are uniformly bounded and therefore the term in the left is uniformly bounded, as well.

We summarize this in the following lemma. 
Lemma 9. There are constants $C>0, \varepsilon_{0}>0$ such that, for every $\varepsilon \leq \varepsilon_{0}$,

$$
\left\|v_{x x}^{(\varepsilon)}\right\|_{L^{\infty}[D]}+\left\|v_{x}^{(\varepsilon)}\right\|_{L^{\infty}[D]}+\left\|v_{t}^{(\varepsilon)}\right\|_{L^{\infty}[D]}+\left\|v^{(\varepsilon)}\right\|_{L^{\infty}[D]} \leq C .
$$

We now obtain the following (see [7]).

Proposition 10. For any $1<p<\infty$ and $t \in\left[0, T^{\prime}\right], v^{(\varepsilon)} \rightarrow$ $v$ as $\varepsilon \rightarrow 0$ weakly in $W^{1, p}(D)$. Furthermore, $v^{(\varepsilon)} \rightarrow v$ uniformly on $D$ and also $v_{x}^{(\varepsilon)} \rightarrow v_{x}$ uniformly in $x \in[0, K]$ for each $t \in\left[0, T^{\prime}\right]$. The function $v$ is the unique solution of $v . i$. Problem 1.

Next, we analyze properties of second order derivatives starting with the following result.

Lemma 11. There is a constant $C>0$ such that, for any $0<$ $\varepsilon \leq \varepsilon_{0}$

$$
\int_{0}^{T^{\prime}} \int_{a_{0}}^{b}\left(v_{t x}^{(\varepsilon)}(t, x)\right)^{2} d x d t<C .
$$

Proof. Set $v=v^{(\varepsilon)}, \beta=\beta^{(\varepsilon)}$, and $w=v_{t}^{(\varepsilon)}$. Multiply (48) by $w$ to obtain

$$
w w_{t}-\frac{\kappa^{2}}{2} w w_{x x}-\mu w w_{x}+\left(r+r K \beta^{\prime}(v)\right) w^{2}=0 .
$$

Integrating this equation over $\left(a_{0}, b\right)$ and recalling that $\beta^{\prime}(v), t$ and $K$ are nonnegative we obtain that, for any $0 \leq$ $t \leq T^{\prime}$

$$
\begin{gathered}
\frac{1}{2} \frac{d}{d t} \int_{a_{0}}^{b} w^{2}(t, x) d x-\frac{\kappa^{2}}{2} \int_{a_{0}}^{b} w(t, x) w_{x x}(t, x) d x \\
\quad-\mu \int_{a_{0}}^{b} \frac{1}{2} \frac{d w^{2}}{d x}(t, x) d x \leq 0 .
\end{gathered}
$$

By (47) and (55) we estimate the third term in (68):

$$
\begin{aligned}
\left|\mu \int_{a_{0}}^{b} \frac{1}{2} \frac{d w^{2}}{d x}(t, x) d x\right| & =|\mu|\left|w^{2}(t, b)-w^{2}\left(t, a_{0}\right)\right| \\
& =|\mu| w^{2}(t, b)<C_{1}^{2} .
\end{aligned}
$$

For the second term in (68) we see that

$$
\begin{gathered}
-\frac{\kappa^{2}}{2} \int_{a_{0}}^{b} w(t, x) w_{x x}(t, x) d x \\
=\frac{\kappa^{2}}{2}\left(\int_{a_{0}}^{b} w_{x}^{2}(t, x) d x-w(t, b) w_{x}(t, b)\right. \\
\left.+w\left(t, a_{0}\right) w_{x}\left(t, a_{0}\right)\right) .
\end{gathered}
$$

Since $w\left(t, a_{0}\right)=0$ and the function $w(t, x)$ is uniformly bounded in $D$ we see in view of (61) that $w_{x}=v_{t x}^{(\varepsilon)}$ is uniformly bounded near the boundary $\left[0, T^{\prime}\right] \times\{b\}$ and $w\left(t, a_{0}\right) w_{x}\left(t, a_{0}\right)=0$, while $\left|w(t, b) w_{x}(t, b)\right|<C_{2}$ for some constant $C_{2}>0$ independent of $\varepsilon$. Thus, we conclude from (68) that

$$
\frac{1}{2} \frac{d}{d t} \int_{a_{0}}^{b} w^{2}(t, x) d x+\frac{\kappa^{2}}{2} \int_{a_{0}}^{b} w_{x}^{2}(t, x) d x \leq C_{3}
$$

for some $C_{3}>0$ independent of $\varepsilon$. Integrating the last equation over $\left[0, T^{\prime}\right]$ we obtain

$$
\begin{aligned}
& \frac{\kappa^{2}}{2} \int_{0}^{T^{\prime}} \int_{a_{0}}^{b} w_{x}^{2}(t, x) d x d t \\
& \quad+\frac{1}{2} \int_{a_{0}}^{b}\left(w^{2}(T, x)-w^{2}(0, x)\right) d x \leq C_{3} .
\end{aligned}
$$

Since the function $w$ is uniformly bounded it follows that there is $C>0$ independent of $\varepsilon$ such that

$$
\int_{0}^{T^{\prime}} \int_{a_{0}}^{b} w_{x}^{2}(t, x) d x d t \leq C
$$

We will now deal with the $L^{2}$ properties of the function $v_{t t}^{(\varepsilon)}(t, x)$

Lemma 12. There is a constant $C>0$ such that for any $0<$ $\varepsilon \leq \varepsilon_{0}$ and every $0<\sigma \leq t \leq T^{\prime}$,

$$
\int_{a_{0}}^{b}\left(v_{t x}^{(\varepsilon)}(t, x)\right)^{2} d x+\int_{\sigma}^{t} \int_{a_{0}}^{b}\left(v_{t t}^{(\varepsilon)}(x, t)\right)^{2} d x d \tau \leq \frac{C}{\sigma} .
$$

Proof. Set $v=v^{(\varepsilon)}, \beta=\beta^{(\varepsilon)}$, and $w=v_{t}^{(\varepsilon)}$. Multiplying (48) by the function $w_{t}$ we have

$$
w_{t}^{2}-\frac{\kappa^{2}}{2} w_{x x} w_{t}-\mu w_{x} w_{t}+\left(r+r K \beta^{\prime}(v)\right) w w_{t}=0
$$

and an integration with respect to $x$ over $\left(a_{0}, b\right)$ yields

$$
\begin{gathered}
\int_{a_{0}}^{b} w_{t}^{2} d x-\frac{\kappa^{2}}{2} \int_{a_{0}}^{b} w_{x x} w_{t} d x-\mu \int_{a_{0}}^{b} w_{x} w_{t} d x \\
+\int_{a_{0}}^{b}\left(r+r K \beta^{\prime}(v)\right) w w_{t} d x=0 .
\end{gathered}
$$

Fix some $t \in[0, T]$. Since $w_{t}\left(t, a_{0}\right)=w\left(t, a_{0}\right)=0$ we see that

$$
\begin{aligned}
& \frac{\kappa^{2}}{2} \int_{a_{0}}^{b} w_{x x}(t, x) w_{t}(t, x) d x \\
& \quad=\frac{\kappa^{2}}{2} w_{x}(t, b) w_{t}(t, b)-\frac{\kappa^{2}}{4} \frac{d}{d t} \int_{a_{0}}^{b} w_{x}(t, x)^{2} d x .
\end{aligned}
$$

From (61) it follows that $\left(\kappa^{2} / 2\right)\left|w_{x}(t, b) w_{t}(t, b)\right| \leq C_{1}$ for some constant $C_{1}>0$ independent of $\varepsilon$, and so we obtain

$$
\begin{aligned}
& \frac{\kappa^{2}}{4} \frac{d}{d t} \int_{a_{0}}^{b} w_{x}^{2}(t, x) d x+\int_{a_{0}}^{b} w_{t}^{2} d x \\
& \quad+\int_{a_{0}}^{b}\left(r+r K \beta^{\prime}(v)\right) w(t, x) w_{t}(t, x) d x \\
& \leq \mu \int_{a_{0}}^{b} w_{x}(t, x) w_{t}(t, x) d x+C_{1} .
\end{aligned}
$$


Now we deal with the last term in (76). Since $\beta^{\prime \prime}(v) \leq 0$ and $v, w \geq 0$ we obtain that

$$
\begin{aligned}
\int_{a_{0}}^{b}(r & \left.+r K \beta^{\prime}(v)\right) w w_{t} d x \\
= & \frac{1}{2} \int_{a_{0}}^{b}\left(r+r K \beta^{\prime}(v)\right) \frac{d}{d t} w^{2} d x \\
= & \frac{1}{2} \frac{d}{d t} \int_{a_{0}}^{b}\left(r+r K \beta^{\prime}(v)\right) w^{2}(t, x) d x \\
& -\frac{1}{2} \int_{a_{0}}^{b} r K \beta^{\prime \prime}(v) w^{3} d x \\
\geq & \frac{1}{2} \frac{d}{d t} \int_{a_{0}}^{b}\left(r+r K \beta^{\prime}(v)\right) w^{2} d x .
\end{aligned}
$$

We plug this inequality into (78) and obtain

$$
\begin{aligned}
& \frac{1}{2} \frac{d}{d t} \int_{a_{0}}^{b}\left[\frac{\kappa^{2}}{2} w_{x}^{2}(t, x)+\left(r+r K \beta^{\prime}(v)\right) w^{2}(t, x)\right] d x \\
&+\int_{a_{0}}^{b} w_{t}^{2}(t, x) d x \leq \mu \int_{a_{0}}^{b} w_{x}(t, x) w_{t}(t, x) d x+C_{1} .
\end{aligned}
$$

Integrate the last inequality with respect to $\tau^{\prime}$ over the interval $(\tau, t)$ to obtain

$$
\begin{aligned}
& \frac{1}{2} \int_{a_{0}}^{b} {\left[\frac{\kappa^{2}}{2} w_{x}^{2}(t, x)+\left(r+r K \beta^{\prime}(v)\right) w^{2}(t, x)\right] d x } \\
& \quad+\int_{\tau}^{t} \int_{a_{0}}^{b} w_{t}^{2}(t, x) d x d \tau^{\prime} \\
& \leq \mu \int_{\tau}^{t} \int_{a_{0}}^{b}\left|w_{x}(t, x)\right|\left|w_{t}(t, x)\right| d x d \tau^{\prime}+C_{1}(t-\tau) \\
& \quad+\frac{1}{2} \int_{a_{0}}^{b}\left[\frac{\kappa^{2}}{2} w_{x}^{2}(\tau, x)+\left(r+r K \beta^{\prime}(v)\right) w^{2}(\tau, x)\right] d x .
\end{aligned}
$$

Next, integrating in $\tau$ over the interval $(0, \sigma)$ for some $0<$ $\sigma<t$ and taking into account that $\left(r+r K \beta^{\prime}(v)\right) w^{2} \geq 0$ by the property (2) of $\beta$ we obtain that

$$
\begin{aligned}
& \frac{\sigma}{2} \int_{a_{0}}^{b} \frac{\kappa^{2}}{2} w_{x}^{2}(t, x) d x+\int_{0}^{\sigma} \int_{\tau}^{t} \int_{a_{0}}^{b} w_{t}^{2}(t, x) d x d \tau^{\prime} d \tau \\
& \leq C_{2}+|\mu| \int_{0}^{\sigma} \int_{\tau}^{t} \int_{a_{0}}^{b}\left|w_{x}(t, x)\right|\left|w_{t}(t, x)\right| d x d \tau^{\prime} d \tau \\
& \quad+\frac{1}{2} \int_{0}^{\sigma} \int_{a_{0}}^{b}\left[\frac{\kappa^{2}}{2} w_{x}^{2}(\tau, x)+\left(r+r K \beta^{\prime}(v)\right) w^{2}(\tau, x)\right] d x d \tau .
\end{aligned}
$$

Now, by (55), (56), and Lemma 11 together with the CauchySchwarz inequality we estimate the right hand side of (82) by a constant $C_{3}>0$ independent of $\varepsilon$. Hence,

$$
\begin{gathered}
C_{3} \geq \frac{\sigma \kappa^{2}}{4} \int_{a_{0}}^{b} w_{x}^{2} d x+\int_{0}^{\sigma} \int_{\tau}^{t} \int_{a_{0}}^{b} w_{t}^{2} d x d \tau^{\prime} d \tau \\
\geq \frac{\sigma \kappa^{2}}{4} \int_{a_{0}}^{b} w_{x}^{2} d x+\sigma \int_{\sigma}^{t} \int_{a_{0}}^{b} w_{t}^{2} d x d \tau^{\prime}
\end{gathered}
$$

and Lemma 12 follows.

As a corollary of previous results we obtain the following.

Proposition 13. Let $\beta<\sigma<T$ and $a<s(0)<s(\sigma)<b<$ $\ln K$. Define $D^{\sigma}=(0, \sigma) \times(a, b)$. Then

$$
P(t, x) \in H^{2}\left(D^{\sigma}\right),
$$

where by definition $H^{2}[U]$ is the set of all the functions in $L^{2}[U]$ with an $L^{2}$ weak second order derivatives. Also there exists $C>$ 0 such that for every $0 \leq t \leq T^{\prime}$,

$$
\int_{a}^{b}\left|\frac{\partial^{2} P}{\partial x \partial t}(t, x)\right|^{2} d x=\left\|\frac{\partial^{2} P}{\partial x \partial t}(t, x)\right\|_{L^{2}[a, b]}<C .
$$

Proof. From Lemmas 12, 11, and 9 we obtain that $\left\{v^{(\varepsilon)}\right\}_{\varepsilon<\varepsilon_{0}}$ are uniformly bounded in $H^{2}\left[D^{\sigma}\right]$ and so they have a weak limit $\widetilde{v} \in H^{2}\left[D^{\sigma}\right]$. Since $v^{(\varepsilon)} \rightarrow v$ uniformly we must have that $v=\widetilde{v}$, and so $v \in H^{2}\left[D^{\sigma}\right]$. Since $v$ is the solution of (36) we can apply Proposition 7 and using the fact that the constant $C$ in (26) does not depend on $t$ we can obtain in a similar way that for a fixed $\sigma$ there is a constant $C>0$ such that, for every $0 \leq t \leq \sigma$,

$$
\left\|v_{x, t}(t, \cdot)\right\|_{L^{2}\left[a_{0}, b\right]}<C .
$$

From (32) we can deduce the same result for the function $P(t, x)$.

Corollary 14. For each $0 \leq t<T$ the function $v_{t}(t, x)$ is Hölder continuous with the exponent $1 / 2$.

Proof. For every $0<t<T$ Proposition 13 gives us that $v_{t}(t, x) \in H^{1}\left[a_{0}, b\right]$. Hence, the result is a consequence of the Sobolev inequality.

Corollary 15. For every $0 \leq t<T^{\prime}$ the functions $P_{t}(t, x)$ and $P_{x x}(t, x)$ as functions of $x$ are continuous in the closed interval $[s(t), b]$.

Proof. For the function $P_{t}(t, x)$ the result follows from (32) and the previous corollary. Since $P(t, x)$ is a solution of (29) in the interval $\{(t, x): s(t)<x<\ln K\}$ and since the functions $P_{x}(t, x)$ and $P(t, x)$ are continuous in the interval $[s(t), b]$ we obtain the result for $P_{x x}$, as well.

Corollary 16. Let $\beta<\sigma<T$ and $a<s(0)<s(\sigma)<b<\ln K$. Define $E=\{(t, x): 0<t<\beta, a-\mu t<x<b-\mu t\}$ and $u(t, x)=e^{-r t} P(t, x+\mu t)$. Then

$$
u(t, x) \in L^{2}[E],
$$


and there exists $C>0$ such that, for every $0 \leq t \leq \beta$,

$$
\int_{a-\mu t}^{b-\mu t}\left|\frac{\partial^{2} u}{\partial x \partial t}(t, x)\right|^{2} d x<C
$$

Proof. The assertion (87) follows from Proposition 13 and the definition of $u(t, x)$. For $(88)$ note that

$$
\begin{gathered}
\frac{\partial^{2} u}{\partial x \partial t}(t, x)=e^{-r t}\left(-r \frac{\partial P}{\partial x}(t, x+\mu t)+\mu \frac{\partial^{2} P}{\partial x^{2}}(t, x+\mu t)\right. \\
\left.+\frac{\partial^{2} P}{\partial x \partial t}(t, x+\mu t)\right) ;
\end{gathered}
$$

then use (85) and the fact that for $(t, x) \in E$ the functions $\left(\partial^{2} P / \partial x^{2}\right)(t, x+\mu t)$ and $(\partial P / \partial x)(t, x+\mu t)$ are bounded.

\section{Price Function Near the Writer's Exercise Boundary}

4.1. Regularity Properties of Price Function. Let $F(t, x)$ be the price function of the put game option (see Section 2). We begin this section by showing that near the writer's exercise region $\Gamma_{1}=\{(t, K): 0 \leq t \leq \beta\}$ the function $\partial F / \partial t$ is continuous. Let

$$
Y_{t}^{[s, x]}=\left(Y_{t}^{1,[s, x]}, Y_{t}^{2,[s, x]}\right)=\left(s+t, S_{t}^{x}\right),
$$

which is a non homogeneous in time Markov process in $\mathbb{R}^{+} \times$ $\mathbb{R}$, where $S_{t}^{x}=x e^{\mu t+\kappa B_{t}}$ and $\mu=r-\left(\kappa^{2} / 2\right)$. Let

$$
\mathbf{L}_{Y}=\frac{\partial}{\partial t}+\frac{\kappa^{2} x^{2}}{2} \frac{\partial^{2}}{\partial x^{2}}+r x \frac{\partial}{\partial x}-r,
$$

which is the infinitesimal generator of $Y_{t}$ when considered on the space of all $C^{2}$ functions. This is a parabolic operator with bounded smooth coefficients in the domain

$$
D=(0, \beta) \times(k, K),
$$

where $k>0$. Let $\mathbf{P}_{[s, x]}$ and $\mathbf{E}_{[s, x]}$ be the probability and the corresponding expectation for the Markov process $Y$ starting at the point $[s, x]$. We will first show that for any $t_{0} \in[0, \beta)$,

$$
\lim _{(t, x) \rightarrow\left(t_{0}, K\right)} \mathbf{P}_{[t, x]}\left[Y_{\tau} \in \Gamma_{1}\right]=1,
$$

where $\tau=\tau(\Gamma)$ and for any closed set $Q \subset \mathbb{R}_{+} \times \mathbb{R}$ we set $\tau(Q)$ to be the arrival time at the set $Q$ for a Markov process under consideration which is $Y_{t}$ here. Indeed, choosing an appropriate nonnegative function $\phi \leq 1$ on the boundary $\Gamma$ and relying on Chapter 3 in [14] we can choose $u(t, x) \epsilon$ $C^{1,2}(D)$ which solves the equation $\mathbf{L}_{Y} u=0$ in $D$ and equals 1 on the boundary part $\Gamma_{1}$ for $0 \leq t \leq t_{1}<\beta$ while decaying smoothly to 0 when $t$ grows to $\beta$. Then

$$
u(t, x)=\mathbf{E}_{[t, x]} \phi\left(Y_{\tau}\right) \leq \mathbf{P}_{[t, x]}\left\{Y_{\tau} \in \Gamma_{1}\right\},
$$

and so

$$
\begin{aligned}
1 & \geq \liminf _{(t, x) \rightarrow\left(t_{0}, K\right)} \mathbf{P}_{[t, x]}\left[Y_{\tau} \in \Gamma_{1}\right] \\
& \geq \lim _{(t, x) \rightarrow\left(t_{0}, K\right)} u(t, x)=u\left(t_{0}, K\right)=1 .
\end{aligned}
$$

Next let $f(x)=(K-x)^{+}$and $g(x)=f(x)+\delta$. Recall that the price of a put game option with an expiration time $T$ and a constant penalty $\delta$ can be written in the form

$$
F(t, x)=\sup _{0 \leq \tau \leq \widetilde{T}} \inf _{0 \leq \sigma \leq \widetilde{T}} J_{[t, x]}(f, g, \sigma, \tau),
$$

where $\widetilde{T}=\inf \left\{t: Y_{t}^{1}=T\right\}$ and for any bounded Borel functions $\widehat{f}$ and $\widehat{g}$ we write

$$
\begin{aligned}
J_{[t, x]} & (\widehat{f}, \widehat{g}, \sigma, \tau) \\
& =\mathbf{E}_{[t, x]}\left[e^{-r \sigma \wedge \tau}\left(\widehat{g}\left(Y_{\sigma}^{2}\right) \rrbracket_{\{\sigma<\tau\}}+\widehat{f}\left(Y_{\tau}^{2}\right) \rrbracket_{\{\tau \leq \sigma\}}\right)\right] .
\end{aligned}
$$

Set

$$
\begin{gathered}
f_{s}(x)=F(s, x) \quad \text { when } \beta<s<T, \\
F_{s}(t, x)=\sup _{0 \leq \tau \leq \widetilde{s}} \inf _{0 \leq \sigma \leq \widetilde{s}} J_{[t, x]}\left(f_{s}, g, \sigma, \tau\right),
\end{gathered}
$$

where $\widetilde{s}=\inf \left\{u: Y_{u}^{(1)}=s\right\}$. Let $\left\langle\sigma^{*}, \tau^{*}\right\rangle$ and $\left\langle\sigma_{s}^{*}, \tau_{s}^{*}\right\rangle$ be the two saddle points (see [1]) corresponding to the optimal stopping games with values $F(t, x)$ and $F_{s}(t, x)$, respectively, and so

$$
\begin{aligned}
& \sigma^{*}=\inf \left\{0 \leq t \leq \widetilde{T}: F\left(Y_{t}\right)=g\left(Y_{t}^{2}\right)\right\}, \\
& \tau^{*}=\inf \left\{0 \leq t \leq \widetilde{T}: F\left(Y_{t}\right)=f\left(Y_{t}^{2}\right)\right\}, \\
& \sigma_{s}^{*}=\inf \left\{0 \leq t \leq \widetilde{s}: F_{s}\left(Y_{t}\right)=g\left(Y_{t}^{2}\right)\right\}, \\
& \tau_{s}^{*}=\inf \left\{0 \leq t \leq \widetilde{s}: F_{s}\left(Y_{t}\right)=f_{s}\left(Y_{t}^{2}\right)\right\} .
\end{aligned}
$$

Then

$$
\begin{gathered}
F(t, x)=J_{[t, x]}\left(f, g, \sigma^{*}, \tau^{*}\right), \\
F_{s}(t, x)=J_{[t, x]}^{s}\left(f_{s}, g, \sigma_{s}^{*}, \tau_{s}^{*}\right) .
\end{gathered}
$$

Lemma 17. For all $0 \leq t \leq s<T$ and $x>0, F_{s}(t, x)=F(t, x)$.

Proof. We have

$$
\begin{aligned}
F_{s}(t, x)= & J_{[t, x]}\left(f_{s}, g, \sigma_{s}^{*}, \tau_{s}^{*}\right) \leq J_{[t, x]}\left(f_{s}, g, \sigma^{*}, \tau_{s}^{*}\right) \\
= & \mathbf{E}_{[t, x]}\left[e ^ { - r \sigma ^ { * } \wedge \tau _ { s } ^ { * } } \left(f_{s}\left(Y_{\tau_{s}^{*}}^{2}\right) \mathbb{q}_{\left\{\tau_{s}^{*} \leq \sigma^{*}\right\}}\right.\right. \\
& \left.\left.+F\left(Y_{\sigma^{*}}\right) \mathbb{q}_{\left\{\sigma^{*}<\tau_{s}^{*}\right\}}\right)\right] \\
\leq & \mathbf{E}_{[t, x]}\left[e ^ { - r \sigma ^ { * } \wedge \tau _ { s } ^ { * } } \left(F\left(Y_{\tau_{s}^{*}}\right) \mathbb{q}_{\left\{\tau_{s}^{*} \leq \sigma^{*}\right\}}\right.\right. \\
& \left.\left.+F\left(Y_{\sigma^{*}}\right) \mathbb{q}_{\left\{\sigma^{*}<\tau_{s}^{*}\right\}}\right)\right] \\
= & \mathbf{E}_{[t, x]}\left[e^{-r \sigma^{*} \wedge \tau_{s}^{*}} F\left(Y_{\sigma^{*} \wedge \tau_{s}^{*}}\right)\right] \leq F(t, x) .
\end{aligned}
$$


Indeed, the first inequality above follows by the saddle point property. The second inequality holds true since $F$ is nonincreasing in the time variable, $\tau_{s}^{*} \leq \widetilde{s}=s-t$ for $Y^{[t, x]}$ and $Y^{1,[t, x]}\left(\tau_{s}^{*}\right) \leq s$. The third inequality is satisfied since the process $e^{-r Y_{\sigma^{*} \wedge u}^{\mathrm{s}}} F\left(Y_{\sigma^{*} \wedge u}\right)$ is a continuous supermartingale in $u$ with respect to $\mathbf{P}_{[t, x]}$ (see [15]). For the other direction we have

$$
\begin{aligned}
& F(t, x) \leq \mathbf{E}_{[t, x]}\left[e^{-r \widetilde{s} \wedge \sigma_{s}^{*} \wedge \tau^{*}} F\left(Y_{\widetilde{s} \wedge \sigma_{s}^{*} \wedge \tau^{*}}\right)\right] \\
& =\mathbf{E}_{[t, x]}\left[e ^ { - r \tilde { s } \wedge \sigma _ { s } ^ { * } \wedge \tau ^ { * } } \left(f\left(Y_{\tau^{*}}^{2}\right) \rrbracket_{\tau^{*} \leq \widetilde{s} \wedge \sigma_{s}^{*}}+F\left(Y_{\widetilde{s}}\right) \rrbracket_{\widetilde{s}<\tau^{*} \wedge \sigma_{s}^{*}}\right.\right. \\
& \left.\left.+g\left(Y_{\sigma_{s}^{*}}^{2}\right) \rrbracket_{\sigma_{s}^{*}<\tilde{s} \wedge \tau^{*}}\right)\right] \\
& \leq \mathbf{E}_{[t, x]}\left[e ^ { - r \tilde { s } \wedge \sigma _ { s } ^ { * } \wedge \tau ^ { * } } \left(f_{s}\left(Y_{\tau^{*} \wedge s}^{2}\right) \rrbracket_{\tau^{*} \wedge \tilde{s} \leq \sigma_{s}^{*}}\right.\right. \\
& \left.\left.+g\left(Y_{\sigma_{s}^{*}}^{2}\right) \rrbracket_{\sigma_{s}^{*}<\tilde{s} \wedge \tau^{*}}\right)\right] \\
& =J_{[t, x]}\left(f_{s}, g, \sigma_{s}^{*}, \tau^{*} \wedge \widetilde{s}\right) \\
& \leq J_{[t, x]}\left(f_{s}, g, \sigma_{s}^{*}, \tau_{s}^{*}\right)=F_{s}(t, x),
\end{aligned}
$$

where we use the submartingale property of $e^{-r Y_{\tau^{*} \wedge u}^{1}} F\left(Y_{\tau^{*} \wedge u}\right)$ in $u$.

Now for any bounded Borel functions $\widehat{f}$ and $\hat{g}$ set

$$
I_{s}(t, x, \widehat{f}, \widehat{g})=\sup _{0 \leq \tau \leq \widehat{\mathcal{S}}} \inf _{0 \leq \sigma \leq \widehat{\mathcal{S}}} J_{[t, x]}(\widehat{f}, \widehat{g}, \sigma, \tau) .
$$

From the time homogeneity of the process $Y_{t}^{2}=S_{t}$ we obtain that

$$
I_{s+h}(t+h, x, \widehat{f}, g)=I_{s}(t, x, \widehat{f}, \widehat{g}) .
$$

Proposition 18. There is a constant $C>0$ such that for any $(t, x) \in(0, \beta) \times(k, K)$,

$$
0 \leq-\frac{\partial F}{\partial t}(t, x) \leq C \mathbf{P}_{[t, x]}\left[\tau_{s}^{*}<\sigma_{s+h}^{*}\right] .
$$

Proof. The left hand side of the above inequality follows from (iii) and (iv) of Proposition 3. For the right hand side, let $h>0$ be such that $\beta+h<T-h$ and $t+h<\beta$ and let $\beta<s<T-h$. By [3] the price function of an American put option has a bounded derivative with respect to $t$ in $[0, s+h] \times \mathbb{R}$; that is, $C=\sup _{(t, x) \in[0, s+h] \times \mathbb{R}_{+}}\left|\left(\partial F_{A}(t, x)\right) / \partial t\right|<\infty$. This together with Proposition 3(ii) yields

$$
\sup _{\beta<s<T, x \geq 0}\left|\frac{\partial F(s, x)}{\partial s}\right| \leq C .
$$

Next, by Lemma 17 and the saddle point property,

$$
\begin{aligned}
F(t, x) & =F_{s}(t, x) \\
& =J_{[t, x]}\left(f_{s}, g, \sigma_{s}^{*}, \tau_{s}^{*}\right) \leq J_{[t, x]}\left(f_{s}, g, \sigma_{s+h}^{*}, \tau_{s}^{*}\right) .
\end{aligned}
$$

By Lemma 17, (104), and the saddle point property,

$$
\begin{aligned}
F(t+h, x) & =F_{s+h}(t+h, x)=I_{s+h}\left(t+h, x, f_{s+h}, g\right) \\
& =I_{s}\left(t, x, f_{s+h}, g\right)=J_{[t, x]}\left(f_{s+h}, g, \sigma_{s+h}^{*}, \tau_{s+h}^{*}\right) \\
& \leq J_{[t, x]}\left(f_{s+h}, g, \sigma_{s+h}^{*}, \tau_{s}^{*}\right) .
\end{aligned}
$$

Now, (98), (106), (107), and (108) yield that

$$
\begin{aligned}
0 & \leq \frac{1}{h}(F(t, x)-F(t+h, x)) \\
& \leq \frac{1}{h} \mathbf{E}_{[t, x]}\left[e^{-r \sigma_{s+h}^{*} \wedge \tau_{s}^{*}}\left(f_{s}\left(Y_{\tau_{s}^{*}}^{2}\right)-f_{s+h}\left(Y_{\tau_{s}^{*}}^{2}\right)\right) \rrbracket_{\left\{\tau_{s}^{*} \leq \sigma_{s+h}^{*}\right\}}\right] \\
& \leq \frac{1}{h} E\left[e^{-r \sigma_{s+h}^{*} \wedge \tau_{s}^{*}} C h \rrbracket_{\left\{\tau_{s}^{*} \leq \sigma_{s+h}^{*}\right\}}\right] \leq C \mathbf{P}_{[t, x]}\left[\tau_{s}^{*} \leq \sigma_{s+h}^{*}\right] .
\end{aligned}
$$

Passing to the limit as $h \rightarrow 0$ we obtain the result.

Corollary 19. For every $0 \leq t_{0}<\beta, \lim _{(t, x) \rightarrow\left(t_{0}, K\right)}$ $(\partial F / \partial t)(t, x)=0$, and so $\lim _{(t, x) \rightarrow\left(t_{0}, \ln K\right)}(\partial P / \partial t)(t, x)=0$.

Proof. In view of Proposition 18 we only have to show that for every $0 \leq t_{0}<\beta$,

$$
\lim _{(t, x) \rightarrow\left(t_{0}, K\right)} \mathbf{P}_{[t, x]}\left[\tau_{s}^{*} \leq \sigma_{s+h}^{*}\right]=0 .
$$

Let $D$ be as in (92), $\Gamma_{2}=\{(\beta, x): k \leq x \leq K\}$, and $\Gamma_{3}=\{(t, k)$ : $0 \leq t \leq \beta\}$. It follows from the definition of $\tau_{s}^{*}$ and $\sigma_{s+h}^{*}$ that for every $(x, t) \in D$,

$$
\left\{\tau\left(\Gamma_{1}\right)<\tau\left(\Gamma_{2} \cap \Gamma_{3}\right)\right\} \subset\left\{\sigma_{s+h}^{*}<\tau_{s}^{*}\right\} \quad \text { with respect to } \mathbf{P}_{[t, x]} .
$$

From (93) we obtain

$$
\lim _{(t, x) \rightarrow\left(t_{0}, K\right)} \mathbf{P}_{[t, x]}\left[\sigma_{s+h}^{*}<\tau_{s}^{*}\right]=1,
$$

and the result follows.

Next, we deal with functions $P(t, x)=F\left(t, e^{x}\right)$, and so it is natural to consider the domain $D_{0}=(0, \beta) \times(k, \ln K)$ for some positive $k<\ln K$ (which is, essentially, the same domain after the space coordinate change) and let

$$
c=P_{A, t}(\beta, \ln K)=\lim _{x \rightarrow \log K} P_{A, t}(\beta, x) .
$$

Let $v(t, x)$ be a function solving the equation $((\partial / \partial t)+$ A) $v(t, x)=0$ with A defined by (27) and satisfying the boundary conditions

$$
\begin{gathered}
v(t, \ln K)=c, \quad v(t, k)=P_{t}(t, k) \quad \text { for } 0 \leq t \leq \beta, \\
v(\beta, x)=P_{t}(\beta, x) \quad \text { for } k<x<\ln K .
\end{gathered}
$$


Since these boundary conditions are continuous then (see [14]) they are satisfied by a unique solution in $C^{1,2}[D]$ of the above equation. Let $w(t, x)$ be a function on $\bar{D}_{0}$ such that

$$
P_{t}(t, x)=w(t, x)+v(t, x) \quad \forall(t, x) \in \bar{D}_{0} \backslash(\beta, \ln K) .
$$

Thus, $w(t, x) \in C^{1,2}\left[D^{\prime}\right]$ and it satisfies the same parabolic equation in $D_{0}$ as $(\partial P / \partial t)(t, x)$ and $v(t, x)$. Its boundary values are

$$
\begin{gathered}
w(t, \ln K)=-c, \quad w(t, k)=0 \quad \text { for } 0 \leq t \leq \beta, \\
w(\beta, x)=0 \quad \text { for } k<x<\ln K .
\end{gathered}
$$

From the continuity of $v(t, x)$ on $\bar{D}_{0}$ we see that it is bounded there and since $\partial P / \partial t$ is also bounded there we obtain the same result for the function $w$ as for $v$. Hence,

$$
w(t, x), v(t, x) \in C^{1,2}\left[D_{0}\right] \cap L^{\infty}\left[D_{0}\right] .
$$

4.2. Integrability of $w_{t}(t, x)$ and $w_{x}(t, x)$. Now we will analyze the function $w(t, x)$. Let $Z_{t}^{[u, x]}=\left(u+t, X_{t}^{x}\right)$ be the diffusion process in the plane whose infinitesimal generator is equal to $\mathbf{L}_{1}=(\partial / \partial t)+\mathbf{A}$ on the space of $C^{2}$ functions. For each $\varepsilon>0$ define $D_{\varepsilon}=(0, \beta-\varepsilon) \times(k+\varepsilon, \ln K-\varepsilon)$. Let $\Gamma_{\varepsilon}$ be the parabolic boundary of $D_{\varepsilon}$. For every $\varepsilon>0$ which is sufficiently small we can find a smooth function $\bar{w}(t, x)$ with compact support on the plane such that in $\bar{D}_{\varepsilon}$ it is equal to $w(t, x)$. By the Dynkin formula we obtain that for every $(u, x) \in D_{\varepsilon}$,

$$
\mathbf{E}_{[u, x]}\left[\bar{w}\left(Z_{\tau\left(\Gamma_{\varepsilon}\right)}\right)\right]=\bar{w}(u, x)+\mathbf{E}_{[u, x]}\left[\int_{0}^{\tau\left(\Gamma_{\varepsilon}\right)} \mathbf{L}_{1} \bar{w}\left(Z_{s}\right) d s\right],
$$

where $\tau(Q)$ denotes the arrival time to $Q$ by the process $Z_{t}^{[u, x]}$. Note that since $w(t, x)=\bar{w}(t, x)$ for $(t, x) \in \bar{D}_{\varepsilon}$ we can replace $\bar{w}$ by $w$ in the above formula and since $Z_{s}^{[u, x]} \in D_{0}$ for $s \leq \tau$ we obtain that $\mathbf{L}_{1} \bar{w}\left(Z_{s}\right)=0$. It follows that for every $\varepsilon>0$,

$$
w(u, x)=\mathbf{E}_{[u, x]}\left[w\left(Z_{\tau\left(\Gamma_{\varepsilon}\right)}\right)\right] .
$$

Now fix $(u, x) \in D_{0}$ and a continuous path $\omega_{0}$. Let $\mathscr{E}=$ $\left\{Z_{\tau\left(\Gamma_{1 / n}\right)}^{[u, x]}\left(\omega_{0}\right)\right\}_{n_{0}<n} \subset \bar{D}_{0}$, where $n_{0}$ is such that $(u, x) \in D_{1 / n_{0}}$. The sequence of times $\left\{\tau\left(\Gamma_{1 / n}\right)(\omega)\right\}_{n>n_{0}}$ is nondecreasing with respect to $n$ and so it has a limit $\rho \leq T$. Let $\gamma$ be an accumulation point in $\mathscr{E}$; that is, $\lim _{k \rightarrow \infty} Z_{\tau\left(\Gamma_{\left.1 / n_{k}\right)}\right.}^{[u, x]}\left(\omega_{0}\right)=\gamma$ for some subsequence $n_{k}$. Define $d\left(y, \Gamma_{0}\right)=\inf \left\{|y-x|: x \in \Gamma_{0}\right\}$ and note that this function is continuous on $\bar{D}_{0}$ and it is 0 if and only if $y \in \Gamma_{0}$. Since $d\left(Y_{\tau\left(\Gamma_{1 / n_{k}}\right)}^{\left[u,{ }_{1}\right)}\left(\omega_{0}\right), \Gamma_{0}\right) \leq 1 / n_{k}$ for each $k$ we conclude that $\gamma \in \Gamma_{0}$ and since $\lim _{k \rightarrow \infty} \tau\left(\Gamma_{1 / n_{k}}\right)=\rho$ it follows that $Z_{\rho}^{[u, x]}\left(\omega_{0}\right)=\lim _{k \rightarrow \infty} Z_{\tau\left(\Gamma_{1 / n_{k}}\right)}^{[u, x]}\left(\omega_{0}\right)=\gamma$. Hence, $\tau\left(\Gamma_{0}\right)\left(\omega_{0}\right)=\rho$. By the definition $w(t, x)$ is continuous except at the point $(\beta, \ln K)$, but because $\mathbf{P}_{[u, x]}\left[Z_{\tau\left(\Gamma_{0}\right)}=(\beta, \ln K)\right]=$ 0 for every $(u, x) \in D_{0}$ we can ignore paths that reach the point $(\beta, \ln K)$, and so

$$
\lim _{\varepsilon \rightarrow 0} w\left(Z_{\tau\left(\Gamma_{\varepsilon}\right)}^{[u, x]}\right)=w\left(Z_{\tau\left(\Gamma_{0}\right)}^{[u, x]}\right) \quad \mathbf{P}_{[u, x]} \text { a.s. }
$$

Corollary 20. For every $(t, x) \in D_{0}$,

$$
\begin{aligned}
w(t, x) & =\mathbf{E}_{[t, x]}\left[w\left(Z_{\tau\left(\Gamma_{0}\right)}\right)\right]=-c \mathbf{E}_{[t, x]}\left[\mathbb{Q}_{\left\{\tau\left(\Gamma_{01}\right)<\tau\left(\Gamma_{02} \cup \Gamma_{03}\right)\right\}}\right] \\
& =-c \mathbf{P}_{[t, x]}\left[\tau\left(\Gamma_{01}\right)<\tau\left(\Gamma_{02} \cup \Gamma_{03}\right)\right],
\end{aligned}
$$

where $\Gamma_{01}=\{(t, \ln K): 0 \leq t \leq \beta\}, \Gamma_{02}=\{(\beta, x): k \leq x \leq$ $\ln K\}$, and $\Gamma_{03}=\{(t, k): 0 \leq t \leq \beta\}$.

Proof. From (117) we know that the function $w(t, x)$ is bounded and so we can use the Lebesgue bounded convergence theorem and from the boundary conditions on $w(t, x)$ it follows that

$$
\begin{aligned}
w(t, x) & =\lim _{\varepsilon \rightarrow 0} \mathbf{E}_{[t, x]}\left[w\left(Z_{\tau\left(\Gamma_{\varepsilon}\right)}\right)\right]=\mathbf{E}_{[t, x]}\left[\lim _{\varepsilon \rightarrow 0} w\left(Z_{\tau\left(\Gamma_{\varepsilon}\right)}\right)\right] \\
& =\mathbf{E}_{[t, x]}\left[w\left(Z_{\tau\left(\Gamma_{0}\right)}\right)\right]
\end{aligned}
$$

which gives the first equality of the corollary, while the second equality follows from (116) and the third equality is obvious.

Let $(t, x),\left(t^{\prime}, x\right) \in D_{0}$ and assume that $t \leq t^{\prime}$. Then it is not difficult to understand that

$$
\begin{aligned}
\mathbf{P}_{[t, x]} & {\left[\tau\left(\Gamma_{01}\right)<\tau\left(\Gamma_{02} \cup \Gamma_{03}\right)\right] } \\
& \geq \mathbf{P}_{\left[t^{\prime}, x\right]}\left[\tau\left(\Gamma_{01}\right)<\tau\left(\Gamma_{02} \cup \Gamma_{03}\right)\right],
\end{aligned}
$$

and so $w(t, x)$ is nonincreasing in $t$ for every $x$ which implies that

$$
\frac{\partial w}{\partial t}(t, x) \geq 0 \quad \forall(t, x) \in D_{0} .
$$

It is also easy to see that for $0 \leq t<T$ and $0 \leq x \leq x^{\prime} \leq \ln K$,

$$
\begin{aligned}
\mathbf{P}_{[t, x]} & {\left[\tau\left(\Gamma_{01}\right)<\tau\left(\Gamma_{02} \cup \Gamma_{03}\right)\right] } \\
& \leq \mathbf{P}_{\left[t, x^{\prime}\right]}\left[\tau\left(\Gamma_{01}\right)<\tau\left(\Gamma_{02} \cup \Gamma_{03}\right)\right],
\end{aligned}
$$

and so

$$
\frac{\partial w}{\partial x} \leq 0 \quad \forall(t, x) \in D_{0} .
$$

Lemma 21. The functions $w_{t}$ and $w_{x}$ are in $L^{1}\left[D_{0}\right]$.

Proof. We will use (124) in order to prove the result for $w_{t}(t, x)$. The case of $w_{x}(t, x)$ can be proven similarly by using 
(126). Using (116), (124), and the continuity of $w(0, x)$ on $\{0\} \times[k, \ln K]$ we obtain that

$$
\begin{aligned}
\int_{D_{0}}\left|\frac{\partial w}{\partial t}\right| d t d x & =\int_{k}^{\ln K} \int_{0}^{\beta} \frac{\partial w}{\partial t} d t d x \\
& =\lim _{\varepsilon \rightarrow 0} \int_{k}^{\ln K-\varepsilon} \int_{0}^{\beta} \frac{\partial w}{\partial t} d t d x \\
& =\lim _{\varepsilon \rightarrow 0} \int_{k}^{\ln K-\varepsilon}(w(\beta, x)-w(0, x)) d x \\
& =-\lim _{\varepsilon \rightarrow 0} \int_{k}^{\ln K-\varepsilon} w(0, x) d x \\
& =-\int_{k}^{\ln K} w(0, x) d x<\infty .
\end{aligned}
$$

Using (126) in place of (124) the proof of integrability of $w_{x}$ is similar.

4.3. Integrability of $v_{t}(t, x)$ and $v_{x}(t, x)$. We continue this section by analyzing the function $v(t, x)$ solving the equation $\mathbf{L}_{1} v=0$ with the boundary conditions given by (114). Let $C^{1,2}\left[\bar{D}_{0}\right]$ be the set of all functions which have one derivative in $t$ and two derivatives in $x$ both uniformly continuous in $D_{0}$.

Lemma 22. There exists a function $z(t, x) \in C^{1,2}\left[\bar{D}_{0}\right]$ such that

$$
z(t, x)=v(t, x) \quad \forall(t, x) \in \Gamma_{0} .
$$

Proof. Recall that $P_{A, t}(T, x)=P_{t}(T, k)$ for $k \leq x<\ln K$ and note that the functions $P_{A, t}(T, x), P_{A, t}(t, x)$, and $P_{t}(t, k)$ as function of $(t, x)$ belong to the space $C^{1,2}\left[\bar{D}_{0}\right]$. Set

$$
\begin{aligned}
\tilde{z}(t, x)= & \frac{\ln K-x}{\ln K-k}\left(P_{t}(t, k)+P_{A, t}(T, x)-P_{A, t}(T, k)\right) \\
& +\frac{x-k}{\ln K-k} P_{A, t}(t, x) .
\end{aligned}
$$

Then $\tilde{z}(t, x) \in C^{1,2}[\bar{D}]$ since it is a linear combination of functions from this space. We also have

$$
\begin{aligned}
& \begin{aligned}
\widetilde{z}(t, k)= & \frac{\ln K-k}{\ln K-k}\left(P_{t}(t, k)+P_{A, t}(T, k)-P_{A, t}(T, k)\right) \\
& =P_{t}(t, x) \quad \forall 0 \leq t \leq \beta
\end{aligned} \\
& \begin{aligned}
\widetilde{z}(t, \ln K) & =P_{A, t}(t, \ln K)
\end{aligned} \\
& \text { when } 0 \leq t \leq \beta, \quad \forall k \leq x \leq \ln K, \\
& \widetilde{z}(T, x)=\frac{\ln K-x}{\ln K-k}\left(P_{t}(T, k)+P_{A, t}(T, x)-P_{A, t}(T, k)\right) \\
& +\frac{x-k}{\ln K-k} P_{A, t}(T, x)=P_{A, t}(T, x) .
\end{aligned}
$$

Thus, we obtain

$$
z(t, x)=\frac{\ln K-x}{\ln K-k} \widetilde{z}(t, x)+\frac{x-k}{\ln K-k} \widetilde{z}(T, x) \in C^{1,2}[\bar{D}] .
$$

Since

$$
\begin{gathered}
z(t, k)=\widetilde{z}(t, k)=P_{t}(t, x), \\
z(t, \ln K)=\widetilde{z}(T, \ln K)=P_{A, t}(T, \ln K)=c, \\
z(T, x)=\tilde{z}(T, x)=P_{A, t}(T, x),
\end{gathered}
$$

it follows that

$$
z(t, x)=v(t, x) \quad \forall(t, x) \in \Gamma .
$$

Next, define $f(t, x)=-\mathbf{L} z(t, x)$. From Lemma 22 we obtain that $f(x, t)$ is bounded in $D_{0}$ and so it belongs to $L^{p}\left[D_{0}\right]$ for every $1 \leq p \leq \infty$. Set $\widetilde{v}(t, x)=v(t, x)-z(x, t)$ and observe that

$$
\mathbf{L} \widetilde{v}(t, x)=f(t, x), \quad \widetilde{v}(t, x)=0 \quad \forall(t, x) \in \Gamma_{0} .
$$

We conclude that the function $\widetilde{v}(t, x)$ is the unique solution of the following problem (see [16]).

Theorem 23. Let $1 \leq p<\infty$, then for any $f(t, x) \in L^{p}\left[D_{0}\right]$ there exists a unique function $\widetilde{v}$ such that

(i) $\tilde{v} \in L^{p}\left[0, T ; W^{2, p}(0,1)\right] \cap L^{p}\left[0, T ; W_{0}^{1, p}(0,1)\right]$,

(ii) $\partial \widetilde{v} / \partial t \in L^{p}\left[D_{0}\right]$,

(iii) $\mathbf{L} \widetilde{v}(t, x)=f(t, x)$ for every $(t, x) \in D_{0}$,

(iv) $\left.\widetilde{v}\right|_{\Gamma_{0}}=0$.

From assertions (i) and (ii) of Theorem 23 we obtain that the functions $\widetilde{v}_{x}(t, x)$ and $\widetilde{v}_{t}(t, x)$ are both in $L^{p}[D]$ for every $0 \leq p<\infty$ and since $z(t, x) \in C^{1,2}\left[\bar{D}_{0}\right]$ we obtain the following.

Corollary 24. For every $1 \leq p<\infty$ the functions $v_{t}(t, x)$ and $v_{x}(t, x)$ belong to the space $L^{p}\left[D_{0}\right]$.

We can now summarize most of the results of this section as follows.

Proposition 25. Let $s(\beta)<k<\ln K<k^{\prime}$ and define

$$
D_{0}=(0, \beta) \times(k, \ln K), \quad D_{0}^{\prime}=(0, \beta) \times\left(\ln K, k^{\prime}\right) .
$$

Then the function $P_{t}(t, x)$ is continuous at every point in the domain $\bar{D}_{0} \backslash\{(\beta, \ln K)\}$, and there exist two functions $w(t, x)$ and $v(t, x)$ on $D_{0}$ such that

$$
\begin{aligned}
P_{t}(t, x)= & w(t, x) \\
& +v(t, x) \quad \text { for every }(t, x) \in \bar{D}_{0} \backslash\{(\beta, \ln K)\},
\end{aligned}
$$

$$
w(t, x), v(t, x) \in C^{1,2}\left(D_{0}\right) \cup L^{\infty}\left[D_{0}\right],
$$


and both functions are solutions of the parabolic equation $\mathbf{L}_{1} u=0$. Furthermore, $w(t, x)$ is continuous in $D_{0}$ and it satisfies

$$
\begin{gathered}
w(t, \ln K)=P_{A, t}(\beta, \ln K), \quad w(t, b)=0 \quad \text { when } 0 \leq t \leq \beta, \\
w(\beta, x)=0 \quad \text { when } k<x<\ln K,
\end{gathered}
$$$$
w_{t}(t, x), w_{x}(t, x) \in L^{1}[D]
$$

Finally, $v(t, x) \in C(\bar{D})$ and for every $1 \leq p<\infty$,

$$
v_{t}(t, x), v_{x}(t, x) \in L^{p}[D] .
$$

The same decomposition of $P_{t}(t, x)$ with the same properties holds true in the domain $D_{0}^{\prime}$.

Proof. Taking the same functions $v$ and $w$ as in (115) we see that (137) is actually the same as (117) and the fact that both $v$ and $w$ are solution of $\mathbf{L}_{1} u=0$ is clear from their definitions. Next we see that (138) is the same as (116), that (139) is the same as Lemma 21, and that (140) is, in fact, Corollary 24. Observe that we did not use in this section the fact that $k<$ $\ln K$, so all the proofs are also applicable to the case $k^{\prime}>\ln K$ and the domain $D_{0}^{\prime}$.

From (136), (139), and (140), and estimating $P_{x x}$ via other derivatives in view of (29) we obtain the following.

Corollary 26. Let $\widetilde{D}=\{(t, x): 0<t<\beta, k-\mu t<x<$ $\ln K-\mu t\}$ and

$$
u(t, x)=e^{-r t} P(t, x+\mu t) .
$$

Then

$$
\frac{\partial^{2} u}{\partial t^{2}} \in L^{1}[\widetilde{D}]
$$

4.4. Price Function When Initial Stock Price Is Large. Let $F(t, x), P(t, x)$, and $u(t, x)$ be as above. Recall that in the domain $(0, T) \times(\ln K, \infty)$ the function $P(t, x)$ satisfies the equation $\mathbf{L}_{1} P=0$ and it is continuous in the closure of $[0, T] \times[\ln K, \infty)$ and $P(T, x)=\left(K-e^{x}\right)^{+}=0$ for $x>\ln K$. Define

$$
v(t, x)=u\left(T-t, \frac{\kappa}{\sqrt{2}} x+\ln K+|\mu| T\right),
$$

where $u$ is given by $(141)$ and set $G=(0, T) \times(0, \infty)$. It follows from Proposition 25 that

(1) $v(t, x) \in C^{1,2}[G] \cup C[\bar{G}]$,

(2) $v_{x x}(t, x)=v_{t}(t, x)$ for every $(t, x) \in G$,

(3) $v(t, 0)=u(T-t, \ln K+|\mu| T)$ is continuous,

(4) $v(0, x)=0$ for every $x>0$,

(5) $v(t, x)$ is bounded (since $P(t, x)$ is).
Since a bounded solution of the heat equation in $G$ is unique (see [13]) then for every $(t, x) \in G$,

$$
\begin{aligned}
& v(t, x)=-2 \int_{0}^{t} \frac{\partial K}{\partial x}(t-\tau, x) v(\tau, 0) d \tau, \\
& \text { where } K(t, x)=\frac{1}{\sqrt{4 \pi t}} e^{-\left(x^{2} / 4 t\right)},
\end{aligned}
$$

and so

$$
v(t, x)=\frac{1}{\sqrt{4 \pi}} \int_{0}^{t} \frac{x e^{-\left(x^{2} / 4(t-\tau)\right)} v(\tau, 0) d \tau}{(t-\tau)^{3 / 2}} .
$$

Differentiating $v$ we obtain polynomials $Q_{k, n}(s, x)$ such that for all $k, n \in \mathbb{N}$,

$$
\frac{\partial^{k+n} v}{\partial^{n} t \partial^{k} x}(t, x)=\int_{0}^{t} Q_{n, k}\left((t-\tau)^{-1 / 2}, x\right) e^{-x^{2} / 4(t-\tau)} v(\tau, 0) d \tau
$$

If $N$ is large enough and $c>0$ then $\left((t-\tau)^{N} / x^{N}\right) Q_{k, n}((t-$ $\left.\tau)^{-1 / 2}, x\right)$ is a polynomial in $(t-\tau)^{1 / 2}$ and $1 / x$ and it is bounded on $(0, T) \times(c, \infty)$. Since $\sup _{y \geq 0} y^{N} e^{-y}<\infty$ for any $N \in \mathbb{N}$ we can set $y=x^{2} / 4(t-\tau)$ deriving that, for any $N \in \mathbb{N}$ and $(t, x) \in(0, T) \times(c, \infty)$,

$$
\begin{gathered}
\frac{\partial^{k+n} v}{\partial^{n} t \partial^{k} x}(t, x) \\
=\int_{0}^{t} \frac{4^{N}(t-\tau)^{N}}{x^{2 N}} Q_{n, k}\left((t-\tau)^{-1 / 2}, x\right) y^{N} e^{-y} v(\tau, 0) d \tau \\
\leq\left(\frac{4}{x}\right)^{N} \int_{0}^{t}\left(\frac{(t-\tau)^{N}}{x^{N}} Q_{n, k}\left(x,(t-\tau)^{-1 / 2}\right)\right) \\
\times y^{N} e^{-y} v(\tau, 0) d \tau \leq \frac{C}{x^{N}} .
\end{gathered}
$$

For some $C=C(N)>0$. Hence, the following results hold true.

Corollary 27. For any $k, n$ positive integers $k, n$, and $c>0$,

$$
\frac{\partial^{k+n} v(t, x)}{\partial^{k} t \partial^{n} x} \in L^{2}[(0, T) \times(c, \infty)]
$$

Corollary 28. Let $(\sqrt{2} / \kappa)(\ln K+|\mu| T)<k^{\prime}$ and $\widetilde{G}=\{(t, x)$ : $\left.0<t<\beta, k^{\prime}-\mu<x<\infty\right\}$. Then

$$
\frac{\partial^{2} u}{\partial t^{2}}(t, x) \in L^{2}[\widetilde{G}]
$$

\section{Proof of Main Theorem}

We split the proof into two cases for $x \leq \ln K$ and for $x>$ $\ln K$. 
5.1. Case $x \leq \ln K$. We begin by proving the upper bound in (12). Since the option holder can exercise at time 0 it is clear from the definition of $P(t, x)$ in (5) that $P(t, x) \geq \psi(x)$ for every $x>0$. Furthermore, by Proposition 3(iv) for each fixed $t$ the function $P(t, x)$ as a function of $x$ is nonincreasing. Therefore, $P(t, x) \geq P(t, \ln K)=\delta$ when $x \leq \ln K$. From the definition (8) of the stopping time $\sigma^{(n)}$ it is not difficult to see that in the present case when $\sigma^{(n)}<T$,

$$
x+\mu \sigma^{(n)}+\kappa W_{\sigma^{(n)}}<\ln K
$$

and so

$$
P\left(\sigma^{(n)}, x+\mu \sigma^{(n)}+\kappa W_{\sigma^{(n)}}\right) \geq \delta
$$

Hence for every $\tau \in \mathscr{T}^{(n)}$ we obtain

$$
\begin{aligned}
\mathbf{E} & {\left[e^{-r \tau \wedge \sigma^{(n)}}\left(\psi\left(x+\mu t+\kappa W_{\tau}^{(n)}\right) \mathbb{q}_{\tau \leq \sigma^{(n)}}+\delta \rrbracket_{\sigma^{(n)}<\tau}\right)\right] } \\
& \leq \mathbf{E}\left[e^{-r \tau \wedge \sigma^{(n)}} P\left(\tau \wedge \sigma^{(n)}, x+\mu \tau \wedge \sigma^{(n)}+\kappa W_{\tau \wedge \sigma^{(n)}}^{(n)}\right)\right] \\
& =\mathbf{E}\left[u\left(\tau \wedge \sigma^{(n)}, X_{\tau \wedge \sigma^{(n)}}^{(n)}\right)\right] .
\end{aligned}
$$

By Proposition 4,

$$
\begin{aligned}
\mathbf{E} & {\left[u\left(\tau \wedge \sigma^{(n)}, X_{\tau \wedge \sigma^{(n)}}^{(n)}\right)\right] } \\
& =u(0, x)+\mathbf{E}\left[\sum_{j=1}^{h^{-1}\left(\tau \wedge \sigma^{(n)}\right)} \mathscr{D} u\left((j-1) h, X_{(j-1) h}^{(n)}\right)\right],
\end{aligned}
$$

where, as before, $u(t, x)=e^{-r t} P(t, x+\mu t)$. Taking the sup with respect to all $\tau \in \mathscr{T}^{(n)}$ in the inequality (152) and using the fact that $u(0, x)=P(0, x)$ we obtain that

$$
\begin{aligned}
& P_{1}^{(n)}(x)-P(0, x) \\
& \quad \leq \sup _{\tau \in \mathscr{T}^{(n)}} \mathbf{E}\left[\sum_{j=1}^{h^{-1}\left(\tau \wedge \sigma^{(n)}\right)} \mathscr{D u}\left((j-1) h, X_{(j-1) h}^{(n)}\right)\right] .
\end{aligned}
$$

Thus, in order to bound $P_{1}^{(n)}(x)-P(0, x)$ from the above it suffices to find an upper bound of the right hand in (154).

Next, we split the domain $[0, T] \times \mathbb{R}$ into three parts

$$
\begin{gathered}
\mathbf{C}=\{(t, x) \in[0, T-h]: \mu t+x>s(t+h)+|\mu| h+\kappa \sqrt{h}\}, \\
\mathbf{S}=\{(t, x) \in[0, T-h]: \mu t+x \leq s(t)-|\mu| h-\kappa \sqrt{h}\}, \\
\mathbf{B}=\{(t, x) \in[0, T-h] \times \mathbb{R}: s(t)-|\mu| h-\kappa \sqrt{h} \\
\leq \mu t+x \leq s(t+h)+|\mu| h+\kappa \sqrt{h}\} .
\end{gathered}
$$

In order to estimate the right hand side of (154) we split it into three parts according to the domains $\mathbf{C}, \mathbf{S}$, and $\mathbf{B}$; that is,

$$
\begin{array}{r}
\mathbf{E}\left[\sum_{j=1}^{h^{-1}\left(\tau \wedge \sigma^{(n)}\right)} \mathscr{D} u\left((j-1) h, X_{(j-1) h}^{(n)}\right)\right] \\
=\mathbf{E}\left[\sum_{j=1}^{h^{-1}\left(\tau \wedge \sigma^{(n)}\right)} \mathscr{D} u\left((j-1) h, X_{(j-1) h}^{(n)}\right) \rrbracket_{\left((j-1) h, X_{(j-1) h}\right) \in \mathbf{C}}\right] \\
+\mathbf{E}\left[\sum_{j=1}^{h^{-1}\left(\tau \wedge \sigma^{(n)}\right)} \mathscr{D} u\left((j-1) h, X_{(j-1) h}^{(n)}\right) \rrbracket_{\left((j-1) h, X_{(j-1) h}\right) \in \mathbf{S}}\right] \\
+\mathbf{E}\left[\sum_{j=1}^{h^{-1}\left(\tau \wedge \sigma^{(n)}\right)} \mathscr{D u}\left((j-1) h, X_{(j-1) h}^{(n)}\right) \rrbracket_{\left((j-1) h, X_{(j-1) h}\right) \in \mathbf{B}}\right] .
\end{array}
$$

By Proposition 3(ii) after the time $\beta$ the prices of the American and game put options coincide which enables us to conclude that $u(t, x)=e^{-r t} P_{A}(t, x+\mu t)$ for $t \geq \beta$ and that the sets $\mathbf{C}_{t \geq \beta}=\{(t, x) \in \mathbf{C}: t \geq \beta\}, \mathbf{S}_{t \geq \beta}=\{(t, x) \in \mathbf{S}: t \geq \beta\}$, and $\mathbf{B}_{t \geq \beta}=\{(t, x) \in \mathbf{B}: t \geq \beta\}$ are the same as the corresponding parts of the domains $\bar{C}, \bar{S}$, and $\bar{B}$ introduced in [3] for the case of American put options. Therefore, we can use the following results from Sections 4.2 and 4.3 in [3].

Proposition 29. There exists a constant $C>0$ such that, for every $\tau \in \mathscr{T}^{(n)}$,

$$
\begin{gathered}
\mathbf{E}\left[\sum_{j=k_{\beta}}^{(\tau / h) \vee k_{\beta}} \mathscr{D}\left|u\left((j-1) h, X_{(j-1) h}^{(n)}\right)\right| \rrbracket_{\left((j-1) h, X_{(j-1) h}\right) \in \mathbf{C}}\right] \\
\quad \leq C\left(\frac{\sqrt{\ln n}}{n}\right)^{4 / 5}
\end{gathered}
$$

where $k_{\beta}=\min \{k: k h \geq \beta\}$ and

$$
\mathbf{E}\left[\sum_{j=k_{\beta}}^{(\tau / h) \vee k_{\beta}} \mathscr{D u}\left((j-1) h, X_{(j-1) h}^{(n)}\right) \mathbb{q}_{\left((j-1) h, X_{(j-1) h}\right) \in \mathbf{B}}\right] \leq \frac{C}{n^{3 / 4}} .
$$

Observe also that $P(t, x)=K-e^{x}$ in the domain $\mathbf{S}$, and so we can use there Lemma 2 from Section 4 of [3].

Lemma 30. For every $(t, x) \in \mathbf{S}$ one has $\mathscr{D} u(t, x) \leq 0$, and so

$$
\mathbf{E}\left[\sum_{j=1}^{h^{-1}\left(\tau \wedge \sigma^{(n)}\right)} \mathscr{D} u\left((j-1) h, X_{(j-1) h}^{(n)}\right) \rrbracket_{\left((j-1) h, X_{(j-1) h}\right) \in \mathbf{S}}\right] \leq 0 .
$$

Thus, for an upper bound of the right side of (154) we can ignore the second term in the right hand side of (156) and estimate only two remaining terms starting with the first term in the right hand side of (156). 
Proposition 31. There is a constant $C>0$ such that, for all $n \in \mathbb{N}$,

$$
\begin{gathered}
\mathbf{E}\left[\sum_{j=1}^{h^{-1}\left(\tau \wedge \sigma^{(n)}\right)}\left|\mathscr{D u}\left((j-1) h, X_{(j-1) h}^{(n)}\right)\right| \rrbracket_{\left((j-1) h, X_{(j-1) h}\right) \in \mathbf{C}}\right] \\
\leq C n^{-3 / 4} .
\end{gathered}
$$

Proof. We have

$$
\begin{aligned}
& \mathbf{E}\left[\sum_{j=1}^{h^{-1}\left(\tau \wedge \sigma^{(n)}\right)}\left|\mathscr{D} u\left((j-1) h, X_{(j-1) h}^{(n)}\right)\right| \rrbracket_{\left((j-1) h, X_{(j-1) h}\right) \in \mathbf{C}}\right] \\
& =\mathbf{E}\left[\sum_{j=1}^{\left(h^{-1}\left(\tau \wedge \sigma^{(n)}\right)\right) \wedge k_{\beta}}\left|\mathscr{D u}\left((j-1) h, X_{(j-1) h}^{(n)}\right)\right|\right. \\
& \left.\times \rrbracket_{\left((j-1) h, X_{(j-1) h}\right) \in \mathbf{C}}\right] \\
& +\mathbf{E}\left[\sum_{j=k_{\beta}}^{\left(h^{-1}\left(\tau \wedge \sigma^{(n)}\right)\right) \vee k_{\beta}}\left|\mathscr{D u}\left((j-1) h, X_{(j-1) h}^{(n)}\right)\right|\right. \\
& \left.\times \mathbb{q}_{\left((j-1) h, X_{(j-1) h}\right) \in \mathbf{C}}\right] .
\end{aligned}
$$

Proposition 29 provides a bound for the second term in the right hand side of (161), and so it remains to deal only with the first term there. Note that if $j h<\sigma^{(n)} \wedge \beta^{(n)}$ and $\left(j h, X_{j h}^{(n)}\right) \in \mathbf{C}$ then

$$
\begin{aligned}
\widetilde{c}_{1}(j) & =s(j h)-\mu j h+\kappa \sqrt{h} \leq X_{j h}^{(n)} \\
& \leq \ln K-2 \kappa \sqrt{h}-\mu j h=\widetilde{c}_{2}(j),
\end{aligned}
$$

where the equalities above are just definitions of $\widetilde{c}_{1}$ and $\widetilde{c}_{2}$. Observe also that since $x<\ln K$ and $j h<\sigma^{(n)}$ then by the definition of the stopping times $\sigma^{(n)}$ the process $X_{j h}^{(n)}+\mu j h$ does not exceed $\ln K-2 K \sqrt{h}$. By Proposition 5 ,

$$
\begin{array}{r}
\mathscr{D u}(t, x) \\
=\frac{1}{\kappa} \int_{0}^{\sqrt{h}} d y \int_{-\kappa y}^{\kappa y} d z\left(z \frac{\partial^{2} u}{\partial t \partial x}\left(t+y^{2}, x+z\right)\right. \\
\left.+\delta(u)\left(t+y^{2}, x+z\right) z\right) .
\end{array}
$$

Relying on the same computation as in Section 4 of [3] we see that for $(t, x) \in \mathbf{C}$ and $x<\ln K-|\mu| h-\kappa \sqrt{h}$,

$$
|\mathscr{D} u(t, x)| \leq \frac{\sqrt{h}}{\kappa} \int_{t}^{t+h} d s \int_{x-\kappa \sqrt{h}}^{x+\kappa \sqrt{h}} d z\left|\frac{\partial^{2} u}{\partial t^{2}}(s, z)\right| .
$$

Thus, for $0 \leq j<k_{\beta}$,

$$
\begin{aligned}
E & \left(\left|\mathscr{D} u\left(j h, X_{j h}^{(n)}\right)\right| \mathbb{q}_{\left\{\left(j h, X_{j h}^{(n)}\right) \in \mathbf{C}\right\} \cap\left\{j h<\sigma^{(n)}\right\}}\right) \\
\leq & \int_{\widetilde{c}_{1}(j)}^{\widetilde{c}_{2}(j)}|D u(j h, y)| d \mathbf{P}_{X_{j h}}(y) \\
\leq & \int_{\widetilde{c}_{1}(j)}^{\widetilde{c}_{2}(j)}\left(\frac{\sqrt{h}}{2 \kappa} \int_{j h}^{j h+h} d s \int_{y-\kappa}^{y+\kappa} \sqrt{h}\left|\frac{\partial^{2} u}{\partial t^{2}}(s, z)\right| d z\right) d \mathbf{P}_{X_{j h}}(y) \\
= & \frac{\sqrt{h}}{2 \kappa} \int_{j h}^{(j+1) h} d s \int_{\widetilde{c}_{1}(j)+\kappa \sqrt{h}}^{\widetilde{c}_{2}(j)+\kappa \sqrt{h}} d z\left|\frac{\partial^{2} u}{\partial t^{2}}(s, z)\right| \\
& \times \int_{\max \left(\widetilde{c}_{1}(j), z-\kappa \sqrt{h}\right)}^{\min \left(\widetilde{c}_{2}(j), z+\kappa \sqrt{h}\right)} d \mathbf{P}_{X_{j h}}(y) \\
\leq & \frac{\sqrt{h}}{2 \kappa} \int_{j h}^{(j+1) h} d s \int_{\widetilde{c}_{1}(j)-\kappa \sqrt{h}}^{\widetilde{c}_{2}(j)+\kappa \sqrt{h}} d z\left|\frac{\partial^{2} u}{\partial t^{2}}(s, z)\right| \\
& \times \mathbf{P}\left[\left|X_{j h}^{(n)}-z\right| \leq \kappa \sqrt{h}\right] .
\end{aligned}
$$

From (24) we see that there is a constant $C>0$ independent of $j$ and $n$ such that

$$
\mathbf{P}\left[\left|X_{j h}^{(n)}-z\right| \leq \kappa \sqrt{h}\right] \leq \frac{C}{\sqrt{j+1}}
$$

Hence, for $j h<\sigma^{(n)}$,

$$
\begin{aligned}
\mathbf{E}(\mid \mathscr{D} & \left.u\left(j h, X_{j h}^{(n)}\right) \mid \rrbracket_{\left(j h, X_{j h}^{(n)}\right) \in \mathbf{C}} \rrbracket_{j h<\sigma^{(n)}}\right) \\
\leq & \frac{\sqrt{h}}{2 \kappa} \int_{j h}^{(j+1) h} d s \int_{\widetilde{\mathcal{c}}_{1}(j)-\kappa \sqrt{h}}^{\widetilde{c}_{2}(j)+\kappa \sqrt{h}} d z\left|\frac{\partial^{2} u}{\partial t^{2}}(s, z)\right| \frac{C}{\sqrt{j+1}} \\
\quad & \frac{C h}{2 \kappa} \int_{j h}^{(j+1) h} \frac{d s}{\sqrt{h(j+1)}} \int_{\widetilde{\mathcal{c}}_{1}(j)-\kappa \sqrt{h}}^{\widetilde{c}_{2}(j)+\kappa \sqrt{h}} d z\left|\frac{\partial^{2} u}{\partial t^{2}}(s, z)\right| \\
\leq & \frac{C_{1}}{n} \int_{j h}^{(j+1) h} \frac{d s}{\sqrt{s}} \int_{\widetilde{\mathcal{c}}_{1}(j)-\kappa \sqrt{h}}^{\widetilde{c}_{2}(j)+\kappa \sqrt{h}} d z\left|\frac{\partial^{2} u}{\partial t^{2}}(s, z)\right| .
\end{aligned}
$$

Define

$$
c_{1}(t)=s(t)-\mu t, \quad c_{2}(t)=\ln K-\mu t-\kappa \sqrt{h},
$$

where $s(t)=\ln (b(t))$ is the free boundary of the option holder and $b(t)$ was introduced at the beginning of Section 3 . Observe that for every $j$ and any $j h \leq s \leq(j+1) h$,

$$
\widetilde{c}_{1}(j)-\kappa \sqrt{h} \geq c_{1}(s), \quad \widetilde{c}_{2}(j)+\kappa \sqrt{h} \leq c_{2}(s) .
$$


Summing up the above estimates we obtain

$$
\begin{gathered}
\sum_{j=0}^{k_{\beta}-1} \mathbf{E}\left(\left|\mathscr{D} u\left(j h, X_{j h}^{(n)}\right)\right| \mathbb{1}_{\left\{\left(j h, X_{j h}^{(n)}\right) \in \mathbf{C}\right\} \cap\left\{j h<\sigma^{(n)}\right\}}\right) \\
\leq \frac{C_{2}}{n}+\frac{C_{1}}{n} \int_{h}^{\beta} \frac{d s}{\sqrt{s}} \int_{\mathcal{c}_{1}(s)}^{c_{2}(s)} d z\left|\frac{\partial^{2} u}{\partial t^{2}}(s, z)\right| \\
=\frac{C_{2}}{n}+\frac{C_{1}}{n}\left(\int_{h}^{\sqrt{h}} \frac{d s}{\sqrt{s}} \int_{\mathcal{C}_{1}(s)}^{c_{2}(s)} d z\left|\frac{\partial^{2} u}{\partial t^{2}}(s, z)\right|\right. \\
\left.+\int_{\sqrt{h}}^{\beta} \frac{d s}{\sqrt{s}} \int_{\mathcal{c}_{1}(s)}^{c_{2}(s)} d z\left|\frac{\partial^{2} u}{\partial t^{2}}(s, z)\right|\right)
\end{gathered}
$$

where the term $C_{2} / n$ comes from the first term $\mathbf{E}|\mathscr{D} u(0, x)|$ of the sum which can be estimated easily using the fact that $u_{t}(t, x)$ and $u_{x x}(t, x)$ are bounded for small $t$.

Let $G=\left\{(t, x): 0<t<\beta, c_{1}(t)<x<\ln K-\mu t\right\}$ and note that $G \subset E \cup \widetilde{D}$, where $E$ and $\widetilde{D}$ are defined in Corollaries 16 and 26 which imply that $\left(\partial^{2} u / \partial t^{2}\right)(s, z) \in L^{1}[F]$. Hence,

$$
\begin{aligned}
& \int_{\sqrt{h}}^{\beta} \frac{d s}{\sqrt{s}} \int_{\mathcal{C}_{1}(s)}^{c_{2}(s)} d z\left|\frac{\partial^{2} u}{\partial t^{2}}(s, z)\right| \\
& \quad \leq C_{1} n^{1 / 4} \int_{\sqrt{h}}^{\beta} d s \int_{\mathcal{c}_{1}(s)}^{c_{2}(s)} d z\left|\frac{\partial^{2} u}{\partial t^{2}}(s, z)\right| \leq C n^{1 / 4} .
\end{aligned}
$$

Next, we estimate the first integral in brackets in the right hand side of (170). Let $s(\beta)<k<\ln K, k^{\prime}=(\ln K-k) / 2$ and split the integral in question as follows:

$$
\begin{aligned}
\int_{h}^{\sqrt{h}} \frac{d s}{\sqrt{s}} \int_{\mathcal{c}_{1}(s)}^{c_{2}(s)} d z\left|\frac{\partial^{2} u}{\partial t^{2}}(s, z)\right| \\
=\int_{h}^{\sqrt{h}} \frac{d s}{\sqrt{s}} \int_{\mathcal{c}_{1}(s)}^{k^{\prime}-\mu s} d z\left|\frac{\partial^{2} u}{\partial t^{2}}(s, z)\right| \\
+\int_{h}^{\sqrt{h}} \frac{d s}{\sqrt{s}} \int_{k^{\prime}-\mu s}^{c_{2}(s)} d z\left|\frac{\partial^{2} u}{\partial t^{2}}(s, z)\right| .
\end{aligned}
$$

From Corollary 16 we know that the function $\left(\partial^{2} u / \partial t^{2}\right)(s, z)$ is in $L^{2}[\widetilde{E}]$, where

$$
\widetilde{E}=\left\{(s, z): 0<s<T, c_{1}(t)<z<k^{\prime}-\mu t\right\} \subset E^{\sigma}
$$

(for an appropriate $b<\ln K$ in the definition of $E^{\sigma}$ ). Therefore we can use the Cauchy-Schwarz inequality to obtain

$$
\begin{aligned}
& \int_{h}^{\sqrt{h}} \frac{d s}{\sqrt{s}} \int_{\mathcal{c}_{1}(s)}^{k^{\prime}-\mu s} d z\left|\frac{\partial^{2} u}{\partial t^{2}}(s, z)\right| \\
& \quad \leq\left(\int_{h}^{\sqrt{h}} \frac{d s}{s} \int_{\mathcal{c}_{1}(s)}^{k^{\prime}-\mu s} d z\right)^{1 / 2}\left(\int_{h}^{\sqrt{h}} \int_{\mathcal{c}_{1}(s)}^{k^{\prime}-\mu s}\left|\frac{\partial^{2} u}{\partial t^{2}}(s, z)\right|^{2} d z\right)^{1 / 2} \\
& \quad \leq C \ln n .
\end{aligned}
$$

Now we are left with the second integral in the right hand side of (172). We will show that there is a constant $C>0$ such that

$$
\mathscr{I}_{n}=\int_{h}^{\sqrt{h}} \frac{d s}{\sqrt{s}} \int_{k^{\prime}-\mu s}^{c_{2}(s)} d z\left|\frac{\partial^{2} u}{\partial t^{2}}(s, z)\right| \leq C n^{1 / 4}
$$

Recall that $u(t, x)=e^{-r t} P(t, x+\mu t)$, and so

$$
\begin{aligned}
& \frac{\partial^{2} u}{\partial t^{2}}(t, x) \\
& =e^{-r t}\left(r^{2} P(t, x+\mu t)-2 r P_{t}(t, x+\mu t)-2 r \mu P_{x}(t, x+\mu t)\right) \\
& \quad+e^{-r t}\left(\mu^{2} P_{x x}(t, x+\mu t)+2 \mu P_{x t}(t, x+\mu t)\right. \\
& \left.\quad+P_{t t}(t, x+\mu t)\right) .
\end{aligned}
$$

Observe that the functions $P(t, x), P_{x}(t, x), P_{t}(t, x)$, and $P_{x x}(t, x)$ are all bounded for small $t$. Indeed, $P \leq K+\delta$, while $P_{t}$ is bounded in the domain of integration in (175) for small $h$ in view of (115), (117), (136), and (137). Next, $P_{x}$ is bounded by Theorem 8.1 from [8]. Finally, $P_{x x}$ is bounded since in the domain in question $P$ and its first derivatives are bounded and $P$ satisfies the equation $((\partial / \partial t)+\mathbf{A}) P=0$ (see (29)). Therefore, we can write

$$
\begin{array}{r}
\mathscr{I}_{n} \leq \int_{h}^{\sqrt{h}} \frac{d t}{\sqrt{t}} \int_{k^{\prime}-\mu t}^{c_{2}(t)} d x\left(\left|2 \mu e^{-r t} P_{t x}(t, x+\mu t)\right|\right. \\
\left.+\left|e^{-r t} P_{t t}(t, x+\mu t)\right|\right)+C_{1},
\end{array}
$$

for some constant $C_{1}>0$ independent of $n$. Recall that, for $(x, t) \in D=(0, \beta) \times(k, \ln K)$ by Proposition $25, P_{t}(t, x)=$ $v(t, x)+w(t, x)$, where $v_{t}$ and $v_{x}$ belong to $L^{2}[D]$. Hence, expressing $P_{t x}$ and $P_{t t}$ via $v_{t}, w_{t}$ and $v_{x}, w_{x}$ we can estimate the integral (177) containing $v_{t}$ and $v_{x}$ by means of the Cauchy-Schwarz inequality as it was done in (174). Replacing these integrals by $C_{2} \sqrt{\ln n}$ we obtain

$$
\begin{aligned}
\mathscr{I}_{n} \leq \int_{h}^{\sqrt{h}} \frac{d t}{\sqrt{t}} \int_{k^{\prime}-\mu t}^{c_{2}(t)} d x\left(\left|2 \mu e^{-r t} w_{x}(t, x+\mu t)\right|\right. \\
\left.+\left|e^{-r t} w_{t}(t, x+\mu t)\right|\right) \\
+C_{2} \sqrt{\ln n}+C_{1} .
\end{aligned}
$$

By (124) and (126) the functions $w_{t}(t, x)$ and $w_{x}(t, x)$ do not change signs in $D$, and so it follows that

$$
\begin{array}{r}
\int_{h}^{\sqrt{h}} \frac{d t}{\sqrt{t}} \int_{k^{\prime}-\mu t}^{c_{2}(t)}\left(\left|2 \mu e^{-r t} w_{x}(t, x+\mu t)\right|\right. \\
\left.+\left|e^{-r t} w_{t}(t, x+\mu t)\right|\right) d x
\end{array}
$$




$$
\begin{gathered}
=\left|\int_{h}^{\sqrt{h}} \frac{d t}{\sqrt{t}} 2\right| \mu\left|e^{-r t} \int_{k^{\prime}}^{\ln K-\kappa \sqrt{h}} w_{x}(t, x) d x\right| \\
+\left|\int_{h}^{\sqrt{h}} \frac{d t}{\sqrt{t}} \int_{k^{\prime}-\mu t}^{c_{2}(t)} e^{-r t} w_{t}(t, x+\mu t) d x\right| .
\end{gathered}
$$

By Proposition 25, $w(x, t)$ is bounded on $D$, and so the contribution of the first integral in the right hand side of (179) is bounded by a constant and it remains to estimate only the second integral there.

Next, we will need a more explicit representation of the function $w$. Let

$$
\tilde{z}(t, x)=e^{-r t} w(t, x+\mu t) .
$$

Then in the domain $\widetilde{E}=\{(t, x), 0<t<\beta, k-\mu t<x<$ $\ln K-\mu t\}$,

$$
\frac{\kappa^{2}}{2} \widetilde{z}_{x x}(t, x)+\widetilde{z}_{t}(t, x)=0
$$

Define

$$
z(t, x)=\widetilde{z}\left(T-t, \frac{\kappa}{\sqrt{2}} x\right)
$$

and let

E

$$
=\left\{(t, x): 0<t<T, \frac{\sqrt{2}(k-\mu t)}{\kappa}<x<\frac{\sqrt{2}(\ln K-\mu t)}{\kappa}\right\} .
$$

In the domain $E$ the function $z(t, x)$ satisfies the heat equation

$$
z_{x x}(t, x)=z_{t}(t, x) .
$$

If we let

$$
\begin{aligned}
& d_{1}(t)=\frac{\sqrt{2}(k-\mu(T-t))}{\kappa}, \\
& d_{2}(t)=\frac{\sqrt{2}(\ln K-\mu(T-t))}{\kappa},
\end{aligned}
$$

then from the boundary values of $w(t, x)$ we obtain

$$
\begin{gathered}
z(0, x)=0 \quad \text { for } d_{1}(0)<x<d_{2}(0), \\
z\left(t, d_{1}(t)\right)=0, \quad z\left(t, d_{2}(t)\right)=e^{-r(T-t)} \text { for } 0<t \leq T .
\end{gathered}
$$

Note that $z(t, x)$ is a bounded continuous function on the boundaries $\left(t, d_{i}(t)\right), i=1,2,0<t \leq T$ of $E$. Hence, by Chapter 14 of [13] we can represent $z(t, x)$ in the form

$$
\begin{aligned}
z(t, x)= & \int_{0}^{t} \frac{\partial H}{\partial x}\left(x-d_{1}(\tau), t-\tau\right) \phi_{1}(\tau) d \tau \\
& +\int_{0}^{t} \frac{\partial H}{\partial x}\left(x-d_{2}(\tau), t-\tau\right) \phi_{2}(\tau) d \tau,
\end{aligned}
$$

where $H(t, x)=(1 / \sqrt{4 \pi t}) e^{-x^{2} / 4 t}$ is the fundamental solution and the functions $\phi_{i}(t), i=1,2$, are bounded continuous on the interval $(0, T]$. From the definition of $\widetilde{z}$ we see that

$$
\widetilde{z}_{t}(t, x)=-r e^{-r t} w(t, x+\mu t)+e^{-r t} w_{t}(t, x+\mu t) .
$$

Since $w(t, x)$ is bounded then for some constant $C_{1}>0$ independent of $n$,

$$
\begin{aligned}
& \left|\int_{h}^{\sqrt{h}} \frac{d t}{\sqrt{t}} \int_{k^{\prime}-\mu t}^{c_{2}(t)} e^{-r t} w_{t}(t, x+\mu t) d x\right| \\
& \quad \leq\left|\int_{h}^{\sqrt{h}} \frac{d t}{\sqrt{t}} \int_{k^{\prime}-\mu t}^{c_{2}(t)} z_{t}(t, x) d x\right|+C_{1} .
\end{aligned}
$$

From the representation (187) of $z(t, x)$ we obtain that

$$
\begin{aligned}
& \left|\int_{h}^{\sqrt{h}} \frac{d t}{\sqrt{t}} \int_{k^{\prime}-\mu t}^{c_{2}(t)} z_{t}(t, x) d x\right| \\
& \leq \frac{\kappa}{\sqrt{2}} \mid \int_{h}^{\sqrt{h}} \frac{d t}{\sqrt{t}} \int_{(\sqrt{2} / \kappa)\left(k^{\prime}-\mu t\right)}^{(\sqrt{2} / \kappa) c_{2}(t)} \frac{d}{d t} \\
& \quad \times \int_{0}^{T-t} \frac{\partial H}{\partial x}\left(x-d_{1}(\tau), T-t-\tau\right) \phi_{1}(\tau) d \tau d x \mid \\
& +\frac{\kappa}{\sqrt{2}} \mid \int_{h}^{\sqrt{h}} \frac{d t}{\sqrt{t}} \int_{(\sqrt{2} / \kappa)\left(k^{\prime}-\mu t\right)}^{(\sqrt{2} / \kappa) c_{2}(t)} \frac{d}{d t} \\
& \quad \times \int_{0}^{T-t} \frac{\partial H}{\partial x}\left(x-d_{2}(\tau), T-t-\tau\right) \phi_{2}(\tau) d \tau d x \mid .
\end{aligned}
$$

Observe that as long as we keep $x$ or $t$ away from 0 the function $H(x, t)$ is smooth and it has bounded derivatives with bounds depending on the range of $t, x$ and their distance from zero. Next, if $x$ satisfies

$$
\frac{\sqrt{2}}{\kappa}\left(k^{\prime}-\mu t\right)<x<\frac{\sqrt{2}}{\kappa} c_{2}(t)=\frac{\sqrt{2}}{\kappa}(\ln K-\mu t-\kappa \sqrt{h}),
$$

then

$$
\begin{aligned}
k^{\prime}-k & \leq \frac{\sqrt{2}}{\kappa}\left(k^{\prime}-k+\mu(T-t-\tau)\right) \\
& <x-d_{1}(\tau) \quad \text { for } 0<\tau \leq T-t .
\end{aligned}
$$

Since $k^{\prime}>k$ we see that $x-d_{1}(\tau)$ stays away from 0 on the entire interval $(0, T-t]$. It follows from the above that the function

$$
\Phi_{1}(t, x)=\int_{0}^{T-t} \frac{\partial H}{\partial x}\left(x-d_{1}(\tau), T-t-\tau\right) \phi_{1}(\tau) d \tau
$$

has bounded derivatives with respect to $t$ with bounds independent of $n$ in the region $\left\{(t, x): h<t<\sqrt{h},(\sqrt{2} / \kappa)\left(k^{\prime}-\right.\right.$ $\left.\mu t)<x(\sqrt{2} / \kappa) c_{2}(t)\right\}$. We conclude that the first integral in 
the right hand side of (190) is bounded from above by a constant independent of $n$ and it remains to estimate the second integral there.

Set

$$
\Phi_{2}(t, x)=\int_{0}^{T-t} \frac{\partial H}{\partial x}\left(x-d_{2}(\tau), T-t-\tau\right) \phi_{2}(\tau) d \tau .
$$

We see that if

$$
x<\frac{\sqrt{2}}{\kappa} c_{2}(t)=\frac{\sqrt{2}}{\kappa}(\ln K-\mu t-\sqrt{h}),
$$

then

$$
\begin{aligned}
x-d_{2}(\tau) & =x-\frac{\sqrt{2}}{\kappa}(\ln K-\mu(T-\tau)) \\
& <\frac{\sqrt{2}}{\kappa}(\mu(T-t-\tau)-\sqrt{h}) .
\end{aligned}
$$

In this case $x-d_{2}(\tau)$ can be zero when $\tau \in[0, T-t]$, but this can happen only for a $\tau$ which is at least $\mu^{-1} \sqrt{h}$ apart from $T-t$. Thus, the function $\Phi_{2}$ is smooth with a bounded uniformly continuous derivative with respect to $t$ though this bound may depend on $n$. Nevertheless, we still have the following:

$$
\begin{aligned}
& \frac{\kappa}{\sqrt{2}} \mid \int_{h}^{\sqrt{h}} \frac{d t}{\sqrt{t}} \int_{(\sqrt{2} / \kappa)\left(k^{\prime}-\mu t\right)}^{(\sqrt{2} / \kappa) c_{2}(t)} \frac{d}{d t} \\
& \times\left(\int_{0}^{T-t} \frac{\partial H}{\partial x}\left(x-d_{2}(\tau), T-t-\tau\right) \phi_{2}(\tau) d \tau\right) d x \mid \\
& =\frac{\kappa}{\sqrt{2}} \mid \int_{h}^{\sqrt{h}} \frac{d t}{\sqrt{t}} \frac{d}{d t}\left(\int_{0}^{T-t} \int_{(\sqrt{2} / \kappa)\left(k^{\prime}-\mu t\right)}^{(\sqrt{2} / \kappa) c_{2}(t)} \frac{\partial H}{\partial x}\right. \\
& \left.\times\left(x-d_{2}(\tau), T-t-\tau\right) d x \phi_{2}(\tau) d \tau\right) \\
& =\frac{\kappa}{\sqrt{2}} \mid \int_{h}^{\sqrt{h}} \frac{d t}{\sqrt{t}} \frac{d}{d t}\left(\int _ { 0 } ^ { T - t } \left(H\left(\frac{\sqrt{2}}{\kappa} c_{2}(t)-d_{2}(\tau), T-t-\tau\right)\right.\right. \\
& -H\left(\frac{\sqrt{2}}{\kappa}\left(k^{\prime}-\mu t\right)\right. \\
& \left.\left.-d_{2}(\tau), T-t-\tau\right)\right) \\
& \left.\times \phi_{2}(\tau) d \tau\right) \\
& \leq \frac{\kappa}{\sqrt{2}} \mid \int_{h}^{\sqrt{h}} \frac{d t}{\sqrt{t}} \frac{d}{d t} \\
& \times\left(\int_{0}^{T-t} H\left(\frac{\sqrt{2}}{\kappa} c_{2}(t)-d_{2}(\tau), T-t-\tau\right)\right. \\
& \left.\times \phi_{2}(\tau) d \tau\right)
\end{aligned}
$$

$$
\begin{aligned}
+\frac{\kappa}{\sqrt{2}} \mid \int_{h}^{\sqrt{h}} \frac{d t}{\sqrt{t}} \frac{d}{d t}\left(\int _ { 0 } ^ { T - t } H \left(\frac { \sqrt { 2 } } { \kappa } \left(k^{\prime}-\ln K\right.\right.\right. \\
+\mu(T-t-\tau)), T-t-\tau) \\
\left.\times \phi_{2}(\tau) d \tau\right) \mid
\end{aligned}
$$

We see that in the second term in the right hand side $k^{\prime}-$ $\ln K+\mu(T-t-\tau)$ can take on the value 0 for $\tau \in(0, T-t]$, but then $\tau$ is at least $c=\mu^{-1}\left|k^{\prime}-\ln K\right|$ apart from $T-t$ and now the separation constant $c$ does not depend on $n$. Thus, we can bound the second term there from above by a constant and it remains to estimate the first term which we do as follows:

I

$$
\begin{aligned}
& =\frac{\kappa}{\sqrt{2}} \mid \int_{h}^{\sqrt{h}} \frac{d t}{\sqrt{t}} \\
& \times \frac{d}{d t} \int_{0}^{T-t} H\left(\frac{\sqrt{2}}{\kappa} c_{2}(t)-d_{2}(\tau), T-t-\tau\right) \\
& \times \phi_{2}(\tau) d \tau \\
& \leq C_{2} \int_{h}^{\sqrt{h}} \frac{d t}{\sqrt{t}} \\
& \times \int_{0}^{T-t} \mid \frac{1}{(T-t-\tau)^{3 / 2}} \\
& \times \exp (-(((\sqrt{2} / \kappa)(\mu(T-t-\tau) \\
& \left.\left.-\kappa \sqrt{h}))^{2}\right) \times(4(T-t-\tau))^{-1}\right) \\
& \times \phi_{2}(\tau) \mid d \tau \\
& +C_{2} \int_{h}^{\sqrt{h}} \frac{d t}{\sqrt{t}} \\
& \times \int_{0}^{T-t} \mid \frac{1}{(T-t-\tau)^{1 / 2}} \\
& \times \exp (-(((\sqrt{2} / \kappa)(\mu(T-t-\tau) \\
& \left.\left.-\kappa \sqrt{h}))^{2}\right) \times(4(T-t-\tau))^{-1}\right) \\
& \times \phi_{2}(\tau) \mid d \tau \\
& +C_{2} \int_{h}^{\sqrt{h}} \frac{d t}{\sqrt{t}} \int_{0}^{T-t} \mid \frac{h}{(T-t-\tau)^{5 / 2}} \exp \left(-\frac{\kappa^{2} h}{2(T-t-\tau)}\right)
\end{aligned}
$$




$$
\begin{aligned}
& \times \exp \left(\frac{\mu \sqrt{h}}{\sqrt{2}}-\frac{\mu^{2}}{2 \kappa^{2}}(T-t-\tau)\right) \\
& \times \phi_{2}(\tau) \mid d \tau,
\end{aligned}
$$

where $C_{2}>0$ is a constant independent of $n$. Analyzing the integral with respect to $\tau$ in the second term in the righthand side above by considering different possible values of $T-t-\tau$ we conclude that this integral is bounded by a constant independent of $n$. Next we observe that $\mid \exp ((\mu \sqrt{h} / \sqrt{2})-$ $\left.\left(\mu^{2} / 2 \kappa^{2}\right)(T-t-\tau)\right) \phi_{2}(\tau) \mid$ is also bounded by a constant independent of $n$ too. Hence, we obtain

$$
\begin{aligned}
\mathbf{I} \leq C_{3}+C_{3} \int_{h}^{\sqrt{h}} \frac{d t}{\sqrt{t}} \int_{0}^{T-t} & \frac{1}{(T-t-\tau)^{3 / 2}} \\
& \times \exp \left(-\frac{h}{2(T-t-\tau)}\right) d \tau \\
+C_{3} \int_{h}^{\sqrt{h}} \frac{d t}{\sqrt{t}} \int_{0}^{T-t} \frac{h}{(T-t-\tau)^{5 / 2}} & \times \exp \left(-\frac{h}{2(T-t-\tau)}\right) d \tau
\end{aligned}
$$

for a constant $C_{3}>0$ independent of $n$. Set $\rho=$ $\sqrt{h / 2(T-t-\tau)}$ and note that $d \rho / d \tau=-\sqrt{h} / 2 \sqrt{2}(T-t-\tau)^{3 / 2}$ and $d \rho^{2} / d \tau=-\sqrt{h} / 4(T-t-\tau)^{2}$. We proceed by changing variables arriving at

$$
\begin{aligned}
\mathbf{I} \leq & C_{4}+C_{4} \int_{h}^{\sqrt{h}} \frac{d t}{\sqrt{t}} \int_{\sqrt{h} / \sqrt{2(T-t)}}^{\infty} \frac{1}{\sqrt{h}} e^{-\rho^{2}} d \rho \\
& +C_{4} \int_{h}^{\sqrt{h}} \frac{d t}{\sqrt{t}} \int_{h / 2(T-t)}^{\infty} \frac{1}{\sqrt{h}} \rho e^{-\rho^{2}} d \rho^{2} \\
\leq & C_{4}+C_{5} \frac{1}{\sqrt{h}} \int_{h}^{\sqrt{h}} \frac{d t}{\sqrt{t}} \leq C_{4}+C_{5} 2\left(1+\frac{1}{h^{1 / 4}}\right) \leq C_{6} n^{1 / 4},
\end{aligned}
$$

for some constants $C_{4}, C_{5}, C_{6}>0$ independent of $n$ and (175) follows. Combining (175) and (174) we obtain from (172) that

$$
\int_{h}^{\sqrt{h}} \frac{d s}{\sqrt{s}} \int_{\mathcal{C}_{2}(s)}^{c_{1}(s)} d z\left|\frac{\partial^{2} u}{\partial t^{2}}(s, z)\right| \leq C n^{1 / 4}
$$

Finally, Proposition 29 follows from (201), (170), and (171).

Next, we turn our attention to the domain B. First, we will prove the following result.
Lemma 32. There exists a constant $C>0$ such that for all $n \in \mathbb{N}$,

$$
\begin{gathered}
\mathbf{E}\left[\sum_{j=1}^{h^{-1}\left(\tau \wedge \sigma^{(n)}\right)} \mathscr{D} u\left((j-1) h, X_{(j-1) h}^{(n)}\right) \rrbracket_{\left((j-1) h, X_{(j-1) h}\right) \in \mathbf{B}}\right] \\
\leq C n^{-3 / 4} .
\end{gathered}
$$

Proof. Let $\mathbf{B}_{t<\beta^{(n)}}$ and $\mathbf{B}_{t \geq \beta^{(n)}}$ be the set of all points $(t, x) \in \mathbf{B}$ such that $t<\beta^{(n)}$ and $t \geq \beta^{(n)}$, respectively. We split (202) according to these two regions, namely,

$$
\begin{aligned}
& \mathbf{E}\left[\sum_{j=1}^{h^{-1}\left(\tau \wedge \sigma^{(n)}\right)} \mathscr{D u}\left((j-1) h, X_{(j-1) h}^{(n)}\right) \mathbb{q}_{\left((j-1) h, X_{(j-1) h}\right) \in \mathbf{B}}\right] \\
& =\mathbf{E}\left[\sum_{j=1}^{h^{-1}\left(\tau \wedge \sigma^{(n)}\right)} \mathscr{D} u\left((j-1) h, X_{(j-1) h}^{(n)}\right)\right. \\
& \left.\times \mathbb{q}_{\left((j-1) h, X_{(j-1) h}\right) \in \mathbf{B}_{t<\beta}}\right] \\
& +\mathbf{E}\left[\sum_{j=1}^{h^{-1}\left(\tau \wedge \sigma^{(n)}\right)} \mathscr{D u}\left((j-1) h, X_{(j-1) h}^{(n)}\right)\right. \\
& \left.\times \rrbracket_{\left((j-1) h, X_{(j-1) h}\right) \in \mathbf{B}_{t \geq \beta}(n)}\right] .
\end{aligned}
$$

By Proposition 29 we have that for a constant $C>0$ independent of $n$,

$$
\begin{gathered}
\mathbf{E}\left[\sum_{j=1}^{h^{-1}\left(\tau \wedge \sigma^{(n)}\right)} \mathscr{D u}\left((j-1) h, X_{(j-1) h}^{(n)}\right) \rrbracket_{\left((j-1) h, X_{(j-1) h}\right) \in \mathbf{B}_{t \geq \beta^{(n)}}}\right] \\
\leq C n^{-3 / 4} .
\end{gathered}
$$

Thus, it remains to estimate only the first term in the righthand side. Let $E=\left\{(t, x): 0<t<\beta^{(n)}, a-\mu t<x<b-\mu t\right\}$, where $a<s(0)$ and $s\left(\beta^{(n)}+h\right)+|\mu| h+2 \kappa \sqrt{h}<b<\ln K$. For $n$ large enough we can find such a $b$ because $s(t)$ is continuous and $s\left(\beta^{(n)}\right)<\ln K$. We know from Corollary 16 that $u(t, x) \in$ $H^{2}[E]$. Since $C^{2}[E]$ is dense in this space we can approximate $u(t, x)$ by $C^{2}$ functions to get equality (22) of Proposition 5 for $u(t, x)$, as well. Since $u_{t}(t, x)+\left(\kappa^{2} / 2\right) u_{x x}(t, x) \leq 0$ in the domain $E$ we obtain

$$
\begin{aligned}
\mathscr{D u}(t, x) & \leq \frac{1}{\kappa} \int_{0}^{\sqrt{h}} d y \int_{-\kappa y}^{\kappa y} d z\left(z \frac{\partial^{2} u}{\partial t \partial x}\left(t+y^{2}, x+z\right)\right) \\
& \leq \int_{0}^{\sqrt{h}} y d y \int_{-\kappa \sqrt{y}}^{\kappa \sqrt{y}} d z\left|\frac{\partial^{2} u}{\partial t \partial x}\left(t+y^{2}, x+z\right)\right| \\
& =\frac{1}{2} \int_{0}^{h} d s \int_{-\kappa \sqrt{y}}^{\kappa \sqrt{y}} d z\left|\frac{\partial^{2} u}{\partial t \partial x}(t+s, x+z)\right| .
\end{aligned}
$$


It follows that

$$
\begin{aligned}
& \mathscr{D u}(t, y) \mathbb{\square}_{(t, y) \in \bar{B}} \\
& \quad \leq \frac{1}{2} \int_{t}^{t+h} d s \int_{s(t)-\lambda \sqrt{h}-\mu t}^{s(t+h)+\lambda \sqrt{h}-\mu t} \mathbb{\square}_{|z-y| \leq \kappa} \sqrt{h}\left|\frac{\partial^{2} u}{\partial t \partial x}(s, z)\right| d z,
\end{aligned}
$$

where $\lambda=|\mu|+\kappa$. Hence,

$$
\begin{array}{r}
\mathbf{E}\left[\sum_{j=1}^{h^{-1}\left(\tau \wedge \sigma^{(n)}\right)} \mathscr{D} u\left((j-1) h, X_{(j-1) h}^{(n)}\right) \mathbb{q}_{\left\{\left((j-1) h, X_{(j-1) h}\right) \in \mathbf{B}_{t<\beta}(n)\right\}}\right] \\
\leq \frac{1}{2}\left(\sum_{j=1}^{k_{\beta}} \int_{j h}^{(j+1) h} d \tau \int_{s(j h)-\lambda \sqrt{h}-\mu j h}^{s(j h+h)+\lambda \sqrt{h}-\mu j h} \mathbf{P}\left(\left|X_{j h}^{(n)}-z\right| \leq \kappa \sqrt{h}\right)\right. \\
\left.\times\left|\frac{\partial^{2} u}{\partial t \partial x}(s, z)\right| d z\right)+\frac{C}{n} .
\end{array}
$$

Here $k_{\beta}=\lceil\beta / h\rceil$, and the term $C / n$ is the contribution of $\mathscr{D} u\left(0, X_{0}^{n}\right)=\mathscr{D} u(0, x) \leq C / n$ which holds true from by the definition of the operator $\mathscr{D}$ and boundedness of $u_{t}$ and $u_{x x}$ for small $t$. From Corollary 16 we see that there exists a constant $C_{1}>0$ such that

$$
\int_{a}^{b}\left|\frac{\partial^{2} u}{\partial t \partial x(t, z)}\right|^{2} d z \leq C_{1} \quad \text { when } 0 \leq t \leq \beta^{(n)} .
$$

This together with (24), the Cauchy-Schwarz inequality, and the inequality $1 / \sqrt{\tau} \geq 1 / \sqrt{2 j h}$, which is satisfied when $j \geq 1$ and $j h \leq \tau \leq 2 j h$, yields that

$$
\begin{gathered}
\left.\mathbf{E}\left[\sum_{j=1}^{h^{-1}\left(\tau \wedge \beta^{(n)}\right)} \mathscr{D} u\left((j-1) h, X_{(j-1) h}^{(n)}\right) \mathbb{q}_{\left\{\left((j-1) h, X_{(j-1) h}\right) \in \mathbf{B}_{t<\beta}(n)\right.}\right\}\right] \\
\leq \sqrt{h} C_{2} \sum_{j=1}^{k_{\beta}} \int_{j h}^{(j+1) h} \frac{d \tau}{\sqrt{\tau}}((s(j+1) h)-s(j h)+2 \lambda \sqrt{h})^{1 / 2} \\
+\frac{C_{2}}{n} .
\end{gathered}
$$

From Proposition 6 and Lipschitz continuity of the function $P(t, x)$ in $t \leq \beta^{(n)}$ uniformly in $x \leq \ln K$ (see Theorem 8.1 in [8]) we obtain that for some constant $C_{3}>0$,

$$
\left|s\left(t_{1}\right)-s\left(t_{2}\right)\right| \leq \sqrt{\left|t_{1}-t_{2}\right|} C_{3} \quad \text { whenever } 0 \leq t_{1}, t_{2} \leq \beta^{(n)} .
$$

Hence,

$$
\begin{aligned}
& \mathbf{E}\left[\sum_{j=0}^{h^{-1}\left(\tau \wedge \beta^{(n)}\right)} \mathscr{D} u\left((j-1) h, X_{(j-1) h}^{(n)}\right) \mathbb{q}_{\left\{\left((j-1) h, X_{(j-1) h}\right) \in \mathbf{B}_{t<\beta}(n)\right\}}\right] \\
& \quad \leq \frac{C_{4}}{n^{3 / 4}}
\end{aligned}
$$

By combining the results of Lemma 30, Proposition 31, and Lemma 32 together with (156) we obtain that the upper bound $P_{1}^{(n)}(x)-P(0, x)<C / n^{3 / 4}$ for some constant $C>0$ independent of $n$ and of $x \leq \ln K$.

Next, we will obtain a lower bound for the approximation error $P_{1}^{(n)}(x)-P(0, x)$ when $x \leq \ln K$. Set

$$
\begin{aligned}
& \tau^{(n)} \\
& =\inf \left\{t: \mu\left[\frac{t}{h}\right] h+X_{t}^{(n)}<s\left(\left[\frac{t}{h}\right] h+h\right)+|\mu| h+\kappa \sqrt{h}\right\} .
\end{aligned}
$$

By Proposition 31,

$$
\begin{aligned}
& \mathbf{E}\left[u\left(\tau^{(n)} \wedge \sigma^{(n)}, X_{\tau^{(n)} \wedge \sigma^{(n)}}^{(n)}\right)\right] \\
& =u(0, x)+\mathbf{E}\left[\sum_{j=1}^{\tau^{(n)} \wedge \sigma^{(n)} / h} \mathscr{D} u\left((j-1) h, X_{(j-1) h}^{(n)}\right)\right] \\
& =u(0, x)+\mathbf{E}\left[\sum_{j=1}^{h^{-1}\left(\tau \wedge \sigma^{(n)}\right)} \mid \mathscr{D} u\left((j-1) h, X_{(j-1) h}^{(n)}\right)\right. \\
& \left.\times \mathbb{q}_{\left((j-1) h, X_{(j-1) h}\right) \in \mathbf{C}}\right] \\
& \geq P(0, x)-C n^{-3 / 4} .
\end{aligned}
$$

Set $\alpha=\alpha_{n}=T-\left(1 / n^{2 / 3}\right)$ and let $\tau_{A}^{(n)}$ be defined by (212) with $s$ there replaced by the free boundary $s_{A}$ for the American put option (see Section 2.2 in [3]). Define also $\tau_{\alpha}^{(n)}=\tau^{(n)} \mathbb{\square}_{\left\{\tau^{(n)}+h<\alpha\right\}}+T \mathbb{\square}_{\left\{\tau^{(n)}+h \geq \alpha\right\}}$ and $\tau_{A, \alpha}^{(n)}=\tau_{A}^{(n)} \rrbracket_{\left\{\tau_{A}^{(n)}+h<\alpha\right\}}+$ $T \square_{\left\{\tau_{A}^{(n)}+h \geq \alpha\right\}}$. We will rely on the following estimate from Section 4.5 in [3].

Lemma 33. There exists a constant $C>0$ independent of $n \mathbb{N}$ such that

$$
\left|\mathbf{E}\left[u_{A}\left(\tau_{A}^{(n)}, X_{\tau_{A}^{(n)}}^{(n)}\right)-e^{r \tau_{A, \alpha}^{(n)}} \psi\left(\mu \tau_{A, \alpha}^{(n)}+X_{\tau_{A, \alpha}^{(n)}}\right)\right]\right| \leq \frac{C}{n^{2 / 3}}
$$

where $u_{A}(t, x)=e^{-t r} P_{A}(t, x+\mu t)$ with $P_{A}$ given by (3).

Remark 34. Note that $s_{A}(t)=s(t)$ for $\beta \leq t<T$, and so $\tau_{A}^{(n)} \vee \beta=\tau^{(n)} \vee \beta$ and $\tau_{A, \alpha}^{(n)} \vee \beta=\tau_{\alpha}^{(n)} \vee \beta$.

From now on we assume that $n$ is large enough so that $\beta^{(n)}<\alpha$. From the definition of $P_{1}^{(n)}(x)$ we have

$$
\begin{aligned}
& P_{1}^{(n)}(x) \\
& \geq \mathbf{E}\left[e^{-r \tau_{\alpha}^{(n)} \wedge \sigma^{(n)}}\left(\psi\left(\mu \tau_{\alpha}^{(n)}+X_{\tau_{\alpha}^{(n)}}^{(n)}\right) \mathbb{q}_{\left\{\tau_{\alpha}^{(n)} \leq \sigma^{(n)}\right\}}+\delta \mathbb{q}_{\left\{\sigma^{(n)}<\tau_{\alpha}^{(n)}\right\}}\right)\right] .
\end{aligned}
$$

for some constant $C_{4}>0$ independent of $n$. 
Hence, if we prove that for some constant $C>0$ independent of $n$

$$
\begin{aligned}
\mathbf{J}= & \mid \mathbf{E}\left[u\left(\tau^{(n)} \wedge \sigma^{(n)}, X_{\tau^{(n)} \wedge \sigma^{(n)}}^{(n)}\right)\right] \\
& -\mathbf{E}\left[e^{-r \tau_{\alpha}^{(n)} \wedge \sigma^{(n)}}\left(\psi\left(\mu \tau_{\alpha}^{(n)}+X_{\tau_{\alpha}^{(n)}}^{(n)}\right) \mathbb{q}_{\left\{\tau_{\alpha}^{(n)} \leq \sigma^{(n)}\right\}}+\delta \rrbracket_{\sigma^{(n)}<\tau_{\alpha}^{(n)}}\right)\right] \mid \\
\leq & \frac{C}{\sqrt{n}}
\end{aligned}
$$

then by (215) and (213) we could conclude that

$$
-\frac{C}{\sqrt{n}} \leq P_{1}^{(n)}(x)-P(0, x) .
$$

We split the left hand side of (216) into three parts

$$
\begin{aligned}
& \mathbf{J}=\mathbf{E}\left[\left\{u\left(\tau^{(n)} \wedge \beta^{(n)}, X_{\tau^{(n)} \wedge \beta^{(n)}}^{(n)}\right)\right.\right. \\
& \left.-e^{-r \tau^{(n)} \wedge \beta^{(n)}}\left(\psi\left(\mu \tau^{(n)} \wedge \beta^{(n)}+X_{\tau^{(n)} \wedge \beta^{(n)}}^{(n)}\right)\right)\right\} \\
& \left.\times \mathbb{q}_{\left\{\tau_{\alpha}^{(n)} \leq \sigma^{(n)} \wedge \beta^{(n)}\right\}}\right] \\
& +\mathbf{E}\left[\left\{u\left(\sigma^{(n)}, X_{\sigma^{(n)}}^{(n)}\right)-e^{-r \sigma^{(n)}} \delta\right\} \mathbb{\square}_{\left\{\sigma^{(n)}<\tau_{\alpha}^{(n)}\right\}}\right] \\
& +\mathbf{E}\left[\left\{u\left(\tau^{(n)}, X_{\tau^{(n)}}^{(n)}\right)-e^{-r \tau_{\alpha}^{(n)}}\left(\psi\left(\mu \tau_{\alpha}^{(n)}+X_{\tau_{\alpha}^{(n)}}^{(n)}\right)\right)\right\}\right. \\
& \left.\times \mathbb{Q}_{\left\{\beta^{(n)}<\tau_{\alpha}^{(n)} \leq \sigma^{(n)}\right\}}\right] .
\end{aligned}
$$

This equality is true since $\tau^{(n)}=\tau_{\alpha}^{(n)}=\tau^{(n)} \wedge \beta$ on the set $\tau_{\alpha}^{(n)} \leq \sigma^{(n)} \wedge \beta^{(n)}<\alpha$. We begin with the last term. First note that on the set $\beta^{(n)} \leq \tau_{\alpha}^{(n)} \leq \sigma^{(n)}$ we have, in particular, $\beta^{(n)} \leq$ $\sigma^{(n)}$ and so $\sigma^{(n)}=T$ by Remark 34. In the case $\tau_{\alpha}^{(n)}>\beta^{(n)}$ we have $\tau_{\alpha}^{(n)}=\tau_{A, \alpha}^{(n)}$ and $\tau^{(n)}=\tau_{A}^{(n)}$ and so from Lemma 33 we derive that

$$
\begin{aligned}
& \mid \mathbf{E}\left[\left\{u\left(\tau^{(n)}, X_{\tau^{(n)}}^{(n)}\right)-e^{-r \tau_{\alpha}^{(n)}}\left(\psi\left(\mu \tau_{\alpha}^{(n)}+X_{\tau_{\alpha}^{(n)}}^{(n)}\right)\right)\right\}\right. \\
& \left.\quad \times \mathbb{\mathbb { V }}_{\left\{\beta^{(n)}<\tau_{\alpha}^{(n)} \leq \sigma^{(n)}\right\}}\right] \mid \\
& \leq\left|\mathbf{E}\left[\left\{u\left(\tau_{A}^{(n)}, X_{\tau_{A}^{(n)}}^{(n)}\right)-e^{-r \tau_{A, \alpha}^{(n)}}\left(\psi\left(\mu \tau_{A, \alpha}^{(n)}+X_{\tau_{A, \alpha}^{(n)}}^{(n)}\right)\right)\right\}\right]\right| \\
& \leq \frac{C}{n^{2 / 3}} .
\end{aligned}
$$

Next, we deal with the first term in the right hand side of (218) where $\tau_{\alpha}^{(n)}=\tau^{(n)} \leq \sigma^{(n)} \wedge \beta^{(n)}$. This means that before time $\beta^{(n)}$ the process $X^{(n)}$ is stopped near the boundary $s(t)$ and

$$
\mu \tau^{(n)}+X_{\tau^{(n)}}^{(n)}<s\left(\tau^{(n)}+h\right)+|\mu| h+\sigma \sqrt{h}
$$

By the definition, $u\left(\tau^{(n)}, X_{\tau^{(n)}}^{(n)}\right)=e^{-r \tau^{(n)}} P\left(\tau^{(n)}, X^{(n)}+\mu \tau^{(n)}\right)$. Thus, we have

$$
\begin{aligned}
& \mathbf{E}\left[\left\{u\left(\tau^{(n)} \wedge \beta^{(n)}, X_{\tau^{(n)}}^{(n)}\right)\right.\right. \\
& \left.\quad-e^{-r \tau^{(n)} \wedge \beta^{(n)}}\left(\psi\left(\mu \tau^{(n)} \wedge \beta^{(n)}+X_{\tau^{(n)} \wedge \beta^{(n)}}^{(n)}\right)\right)\right\} \\
& \left.\quad \times \mathbb{q}_{\left\{\tau_{\alpha}^{(n)} \leq \sigma^{(n)} \wedge \beta^{(n)}\right\}}\right] \\
& =\mathbf{E}\left[\left\{e^{-r \tau^{(n)}}\left(P\left(\tau^{(n)}, X^{(n)}+\mu \tau^{(n)}\right)-\psi\left(\mu \tau^{(n)}+X_{\tau^{(n)}}^{(n)}\right)\right)\right\}\right. \\
& \left.\quad \times \mathbb{\mathbb { V }}_{\left\{\tau_{\alpha}^{(n)} \leq \sigma^{(n)} \wedge \beta^{(n)}\right\}}\right] .
\end{aligned}
$$

If $\mu \tau^{(n)}+X_{\tau^{(n)}}^{(n)} \leq s\left(\tau^{(n)}\right)$, then $P\left(\tau^{(n)}, X^{(n)}+\mu \tau^{(n)}\right)-\psi\left(\mu \tau^{(n)}+\right.$ $\left.X_{\tau^{(n)}}^{(n)}\right)=0$, so we can assume that

$$
s\left(\tau^{(n)}\right)<\mu \tau^{(n)}+X_{\tau^{(n)}}^{(n)}<s\left(\tau^{(n)}+h\right)+|\mu| h+\sigma \sqrt{h} .
$$

To continue we need the following lemma.

Lemma 35. There is a constant $C>0$ independent of $n$ such that for every point $(t, x)$ satisfying $s(t) \leq \mu t+x \leq s(t+h)+$ $|\mu| h+\sigma \sqrt{h}$ and $0 \leq t \leq \beta^{(n)}$,

$$
|P(t, \mu t+x)-\psi(\mu t+x)| \leq \frac{C}{n} .
$$

Proof. The function $P(t, x)$ is Lipschitz continuous when $t \leq$ $\beta$ and $0 \leq x \leq \mu \beta+\ln K$ (see [8]), and so

$$
|P(t, \mu t+x)-P(t+h, \mu t+x)| \leq \frac{C}{n}
$$

for some $C>0$ independent of $n$. If $\mu t+x \leq s(t+h)$ then $P(t+h, \mu t+x)=\psi(\mu t+x)$ and we are done. Now assume that $s(t+h)<\mu t+x<s(t+h)+\lambda / \sqrt{n}$, where $\lambda=\sqrt{n}(|\mu| h+\sigma \sqrt{h})$.

From Corollary 15 it follows that for every $t<T$ and $a<$ $\ln K$ the function $P_{x x}(t, x)$ is continuous in $x$ on the closed interval $[s(t), a]$, so we can write

$$
\begin{aligned}
P(t+h, \mu t+x) \\
=P(t+h, s(t+h))+P_{x}(t+h, s(t+h)) \frac{\lambda}{\sqrt{n}} \\
\quad+P_{x x}(t+h, s(t+h)) \frac{\lambda^{2}}{2 n}+\alpha,
\end{aligned}
$$

where $\alpha=\alpha(h)$ satisfies $\lim _{h \rightarrow 0}(\alpha / h)=0$. From the property of smooth fit (see [8]) it follows that $P_{x}(t+h, s(t+h))=$ $\psi_{x}(s(t+h))$, and so for some $C>0$ independent of $n$,

$$
|P(t+h, \mu t+x)-\psi(\mu t+x)| \leq \frac{C}{n} .
$$


Using (222) and the above lemma we obtain

$$
\begin{aligned}
& \mid \mathbf{E}\left[\left\{u\left(\tau^{(n)} \wedge \beta^{(n)}, X_{\tau^{(n)} \wedge \beta^{(n)}}^{(n)}\right)\right.\right. \\
& \left.\quad-e^{-r \tau^{(n)} \wedge \beta^{(n)}}\left(\psi\left(\mu \tau^{(n)} \wedge \beta^{(n)}+X_{\tau^{(n)} \wedge \beta^{(n)}}^{(n)}\right)\right)\right\} \\
& \left.\quad \times \mathbb{\square}_{\left\{\tau_{\alpha}^{(n)} \leq \sigma^{(n)} \wedge \beta^{(n)}\right\}}\right] \mid \leq \frac{C}{n} .
\end{aligned}
$$

Hence, we are done with the first term in the right hand side of (218) and it remains to estimate the second one. Since $\sigma^{(n)}<\tau_{\alpha}^{(n)} \leq T$ the process $X^{(n)}$ is stopped near the writer's boundary. Namely, we have

$$
\ln K-|\mu| h-\sigma \sqrt{h}<\mu \sigma^{(n)}+X_{\sigma^{(n)}}^{(n)} \leq \ln K
$$

Since $P(t, \ln K)=\delta$ when $t \leq \beta, \beta^{(n)}-\beta<h$, and $P$ is Lipschitz continuous (see Theorem 8.1 of [8]) we obtain that

$$
\left|P\left(\sigma^{(n)}, \mu \sigma^{(n)}+X_{\sigma^{(n)}}^{(n)}\right)-\delta\right| \leq \frac{C}{\sqrt{n}}
$$

for some $C>0$ independent of $n$. Hence,

$$
\mathbf{E}\left[\left(u\left(\sigma^{(n)}, X_{\sigma^{(n)}}^{(n)}\right)-e^{-r \sigma^{(n)}} \delta\right) \mathbb{q}_{\left\{\sigma^{(n)}<\tau_{\alpha}^{(n)}\right\}}\right] \leq \frac{C}{\sqrt{n}} .
$$

It follows that there exists $C>0$ independent of $n$ such that, for every $x \leq \ln K$,

$$
-\frac{C}{\sqrt{n}}<P_{1}^{(n)}(x)-P(0, x) .
$$

Next, we will derive a lower bound for the second approximation function $P_{2}^{(n)}(x)$ defined by (11), still assuming that $x \leq \ln K$. According to (213) in order to obtain

$$
P_{2}^{(n)}(x)-P(0, x) \geq-\frac{C}{n^{2 / 3}}
$$

it suffices to show that

$$
\mathbf{E}\left[u\left(\tau^{(n)} \wedge \sigma^{(n)}, X_{\tau^{(n)} \wedge \sigma^{(n)}}^{(n)}\right)\right]-P_{2}^{(n)}(x) \leq \frac{C}{n^{2 / 3}} .
$$

We have

$$
\begin{aligned}
& \mathbf{E}\left[u\left(\tau^{(n)} \wedge \sigma^{(n)}, X_{\tau^{(n)} \wedge \sigma^{(n)}}^{(n)}\right)\right]-P_{2}^{(n)}(x) \\
& \leq \mathbf{E}\left[u\left(\tau^{(n)} \wedge \sigma^{(n)}, X_{\tau^{(n)} \wedge \sigma^{(n)}}^{(n)}\right)-e^{-r \tau_{\alpha}^{(n)} \wedge \sigma^{(n)}}\right. \\
& \times\left(\psi\left(\mu \tau_{\alpha}^{(n)}+X_{\tau_{\alpha}^{(n)}}^{(n)}\right) \mathbb{q}_{\left\{\tau_{\alpha}^{(n)} \leq \sigma^{(n)}\right\}}\right. \\
& \left.\left.+\left(\psi\left(\mu \sigma^{(n)}+X_{\sigma^{(n)}}^{(n)}\right)+\delta\right) \mathbb{\rrbracket}_{\left\{\sigma^{(n)}<\tau_{\alpha}^{(n)}\right\}}\right)\right] \\
& =\mathbf{E}\left[\left\{u\left(\tau^{(n)} \wedge \beta^{(n)}, X_{\tau^{(n)} \wedge \beta^{(n)}}^{(n)}\right)\right.\right. \\
& \left.-e^{-r \tau^{(n)} \wedge \beta^{(n)}}\left(\psi\left(\mu \tau^{(n)} \wedge \beta^{(n)}+X_{\tau^{(n)} \wedge \beta^{(n)}}^{(n)}\right)\right)\right\} \\
& \left.\times \mathbb{q}_{\left\{\tau_{\alpha}^{(n)} \leq \sigma^{(n)} \wedge \beta^{(n)}\right\}}\right] \\
& +\mathbf{E}\left[\left\{u\left(\sigma^{(n)}, X_{\sigma^{(n)}}^{(n)}\right)-e^{-r \sigma^{(n)}}\left(\psi\left(\mu \sigma^{(n)}+X_{\sigma^{(n)}}^{(n)}\right)+\delta\right)\right\}\right. \\
& \left.\times \mathbb{q}_{\left\{\sigma^{(n)}<\tau_{\alpha}^{(n)}\right\}}\right] \\
& +\mathbf{E}\left[\left\{u\left(\tau^{(n)}, X_{\tau^{(n)}}^{(n)}\right)-e^{-r \tau_{\alpha}^{(n)}}\left(\psi\left(\mu \tau_{\alpha}^{(n)}+X_{\tau_{\alpha}^{(n)}}^{(n)}\right)\right)\right\}\right. \\
& \left.\times \mathbb{\{}_{\left\{\beta^{(n)}<\tau_{\alpha}^{(n)} \leq \sigma^{(n)}\right\}}\right] .
\end{aligned}
$$

Indeed, the first inequality is true since $P_{2}^{(n)}(x)$ is defined as the sup on $\tau \in \mathscr{T}^{(n)}$ and we chose a specific one, that is, $\tau_{\alpha}^{(n)}$. The equality is true due to the same reason that (218) holds true. We see that the first term in the right hand side of (234) is the same as the first term in (218) and by (227) it is less than $C / n$ for some constant $C$. The second term is nonpositive because, for every $(t, x)$ we have $P(t, x) \leq \psi(x)+\delta$ and $u(t, x)=e^{-r t} P(t, \mu t+x)$, so we can just remove it from the equation. The last term is the same as the last term of (218) and from Lemma 33 we obtain that this term is less than or equal $C / n^{2 / 3}$ for an appropriate $C$. These arguments yield (233) and hence (232), as well. For the upper bound we already know that $P_{1}^{(n)}(x)-P(0, x) \leq C / n^{3 / 4}$ and from the definition of $P_{1}^{(n)}$ and $P_{2}^{(n)}$ it is not hard to see that $\mid P_{2}^{(n)}-$ $P_{1}^{(n)} \mid \leq C / \sqrt{n}$. It follows from above that there exist $C>0$ such that, for every $x \leq \ln K$,

$$
-\frac{C}{n^{3 / 2}} \leq P_{2}^{(n)}(x)-P(0, x) \leq \frac{C}{\sqrt{n}} .
$$

5.2. Case $x>\ln K$. We begin with the upper bound on $P_{1}^{(n)}$. We will show first that

$$
\begin{aligned}
P_{1}^{(n)}(x) & -P(0, x) \\
& \leq \sup _{\tau \in \mathscr{T}^{(n)}} \mathbf{E}\left[\sum_{j=1}^{h^{-1}\left(\tau \wedge \sigma^{(n)}\right)} \mathscr{D u}\left((j-1) h, X_{(j-1) h}^{(n)}\right)\right] .
\end{aligned}
$$


The proof is similar to the proof of (154), we just have to show that for every $\tau \in \mathscr{T}^{(n)}$,

$$
\begin{aligned}
P(\tau \wedge & \left.\sigma^{(n)}, \mu \tau \wedge \sigma^{(n)}+X_{\tau \wedge \sigma^{(n)}}^{(n)}\right) \\
\geq & \psi\left(\mu \tau+X_{\tau}^{(n)}\right) \mathbb{q}_{\left\{\tau \leq \sigma^{(n)}\right\}} \\
& \quad+(\delta-\operatorname{Ke}(|\mu| h+2 \kappa \sqrt{h})) \mathbb{q}_{\left\{\sigma^{(n)}<\tau\right\}} .
\end{aligned}
$$

On the set $\tau \leq \sigma^{(n)}$ this inequality is clear since $P(t, x) \geq \psi(x)$. For the case $\sigma^{(n)}<\tau$ observe that because $x>\ln K$ we must have

$$
\ln K<\mu \sigma^{(n)}+X_{\sigma^{(n)}}^{(n)}<\ln K+|\mu| h+2 \kappa \sqrt{h} .
$$

By Theorem 8.1 in [8] the right derivative $F_{x}(t, K+)$ at $K$ satisfies $0>F_{x}(t, K+)>-1$ for any $t$, and so $0 \leq F(t, K)-$ $F(t, K+C \lambda) \leq C \lambda$ for each $C>0$ provided $0 \leq \lambda \leq \lambda(C)$ is small enough. Assume $0<\lambda<1$; then $e^{\lambda}-1 \leq \lambda e^{\lambda} \leq \lambda e$. Hence, taking $C=K e$ we have

$$
\begin{aligned}
& P(t, \ln K)-P(t, \ln K+\lambda) \\
& \quad=F(t, K)-F\left(t, K e^{\lambda}\right) \\
& \quad \leq F(t, K)-F(t, K+K e \lambda) \leq K e \lambda .
\end{aligned}
$$

Put $\lambda=|\mu| h+\kappa \sqrt{h}$; then for $\sigma^{(n)}<\tau$ and sufficiently large $n$,

$$
P\left(\sigma^{(n)}, \mu \sigma^{(n)}+X_{\sigma^{(n)}}^{(n)}\right) \geq P\left(\sigma^{(n)}, \ln K+\lambda\right) \geq \delta-K e \lambda .
$$

Hence, we obtain (237) which yields also (234). To bound the right hand side of (236) we split it similarly into the case $x \leq \ln K$ (see (156)) according to the three different regions $\mathbf{C}, \mathbf{B}$, and $\mathbf{S}$. Since our process starts at $x>\ln K$, if $\left((j-1) h, X_{(j-1) h}^{(n)}\right) \in \mathbf{B}$ for some $j$ then this must happen after the time $\beta$, and so we can use (158). The part that belongs to the region $\mathbf{S}$ is nonpositive, so we can ignore it, and so we will be left only with the region $\mathbf{C}$.

Lemma 36. For the discrete process $X_{t}^{(n)}$ such that $X_{0}^{(n)}=x>$ $\ln K$ one has

$$
\begin{gathered}
\mathbf{E}\left[\sum_{j=1}^{h^{-1}\left(\tau \wedge \sigma^{(n)}\right)} \mathscr{D}\left|u\left((j-1) h, X_{(j-1) h}^{(n)}\right)\right| \rrbracket_{\left\{\left((j-1) h, X_{(j-1) h}\right) \in \mathbf{C}\right\}}\right] \\
\leq C n^{-3 / 4} .
\end{gathered}
$$

Proof. It suffices to show that

$$
\begin{aligned}
& \mathbf{E}\left[\sum_{j=1}^{k_{\beta} \wedge\left(\sigma^{(n)} / h\right)} \mathscr{D}\left|u\left((j-1) h, X_{(j-1) h}^{(n)}\right)\right| \mathbb{q}_{\left\{\left((j-1) h, X_{(j-1) h}\right) \in \mathbf{C}\right\}}\right] \\
& \leq C n^{-3 / 4}
\end{aligned}
$$

for some $C>0$ independent of $n$ since after time $\beta^{(n)}$ we come back to the American option case. This is done in the same way as in Proposition 31, and so we provide only a sketch of the proof. Let $c(s)=\ln K-\mu s+\kappa \sqrt{h}$; then similarly to the proof of Proposition 31 we obtain

$$
\begin{aligned}
& \sum_{j=0}^{k_{\beta}-1} E\left(\left|\mathscr{D u}\left(j h, X_{j h}^{(n)}\right)\right| \mathbb{q}_{\left\{\left(j h, X_{j h}^{(n)}\right) \in C\right\} \cap\left\{j h<\sigma^{(n)}\right\}}\right) \\
& \quad \leq \frac{C_{2}}{n}+\frac{C_{1}}{n} \int_{h}^{\beta} \frac{d s}{\sqrt{s}} \int_{c(s)}^{\infty} d z\left|\frac{\partial^{2} u}{\left(\partial t^{2}\right)}(s, z)\right| .
\end{aligned}
$$

Let $(\sqrt{2} / \kappa)(\ln K+|\mu| T)<k^{\prime}$ and split the integral in (243) into two parts

$$
\begin{aligned}
\int_{h}^{\beta} & \frac{d s}{\sqrt{s}} \int_{c(s)}^{\infty} d z\left|\frac{\partial^{2} u}{\partial t^{2}}(s, z)\right| \\
= & \int_{h}^{\beta} \frac{d s}{\sqrt{s}} \int_{c(s)}^{k^{\prime}-\mu s} d z\left|\frac{\partial^{2} u}{\left(\partial t^{2}\right)}(s, z)\right| \\
& +\int_{h}^{\beta} \frac{d s}{\sqrt{s}} \int_{k^{\prime}-\mu s}^{\infty} d z\left|\frac{\partial^{2} u}{\left(\partial t^{2}\right)}(s, z)\right| .
\end{aligned}
$$

Let $E=\left\{(s, z): 0<s<\beta, k^{\prime}-\mu s<z<\infty\right\}$; then by Corollary 28 we see that $\left(\partial^{2} u / \partial t^{2}\right)(s, z) \in L^{2}[E]$, and so we obtain similarly to (174) that, for some constant $C>0$,

$$
\int_{h}^{\beta} \frac{d s}{\sqrt{s}} \int_{k^{\prime}-\mu s}^{\infty} d z\left|\frac{\partial^{2} u}{\left(\partial t^{2}\right)}(s, z)\right|<C \ln n .
$$

In the first integral in the right hand side of (244) we do the same procedure as in (170)-(175) relying on Proposition 25 and deriving that for some constant $C>0$,

$$
\int_{h}^{\beta} \frac{d s}{\sqrt{s}} \int_{c(s)}^{k^{\prime}-\mu s} d z\left|\frac{\partial^{2} u}{\left(\partial t^{2}\right)}(s, z)\right|<C n^{1 / 4} .
$$

Combining (243)-(246) we obtain (242) and complete the proof of the lemma.

The lower bound for $P_{1}^{(n)}(x)-P(0, x)$ when $x>\ln K$ is obtained similarly to the case $x \leq \ln K$. As in that case we use the stopping time $\tau^{(n)}$ from (212) and from the above we see that (213) is true also for the case under consideration. We consider again $\tau_{\alpha}^{(n)}$ defined before Lemma 33 and similarly to (214) obtain that

$$
\begin{gathered}
\mid \mathbf{E}\left[u\left(\tau_{\alpha}^{(n)} \wedge \sigma^{(n)}, X_{\tau_{\alpha}^{(n)} \wedge \sigma^{(n)}}\right)\right] \\
-\mathbf{E}\left[e ^ { - r \tau _ { \alpha } ^ { ( n ) } \wedge \sigma ^ { ( n ) } } \left(\psi\left(\mu \tau_{\alpha}^{(n)}+X_{\tau_{\alpha}^{(n)}}^{(n)}\right) \mathbb{q}_{\left\{\tau_{\alpha}^{(n)} \leq \sigma^{(n)}\right\}}\right.\right. \\
+(\delta-K e(|\mu| \sqrt{h}+\sigma h)) \\
\left.\left.\times \mathbb{q}_{\left\{\sigma^{(n)}<\tau_{\alpha}^{(n)}\right\}}\right)\right] \mid
\end{gathered}
$$

In order to estimate (247) for $x>\ln K$ we only need to split it into two parts, one for $\tau_{\alpha}^{(n)} \leq \sigma^{(n)}$ and the other one for 
$\sigma^{(n)}<\tau_{\alpha}^{(n)}$. This is true in view of the fact that if we begin with $x>\ln K$ and $\tau_{\alpha}^{(n)} \leq \sigma^{(n)}$ then we must have $\beta^{(n)} \leq \tau_{\alpha}^{(n)}$, and so we are back to the American option case and can use Lemma 33 for this case. If $\sigma^{(n)}<\tau_{\alpha}^{(n)}$ then the process $X^{(n)}$ is stopped near the seller's boundary and similar to (230) we can use the Lipschitz property of $P$ to obtain

$$
\begin{gathered}
\mathbf{E}\left[\left(u\left(\sigma^{(n)}, X_{\sigma^{(n)}}^{(n)}\right)-e^{-r \sigma^{(n)}}(\delta-K e(|\mu| h+2 \kappa \sqrt{h}))\right.\right. \\
\left.\left.\times \mathbb{q}_{\left\{\sigma^{(n)}<\tau_{\alpha}^{(n)}\right\}}\right)\right] \leq \frac{C}{\sqrt{n}} .
\end{gathered}
$$

From here we can proceed similar to the case of $x \leq \ln K$ and obtain the lower bound for $P_{1}^{(n)}$ proving (12) for $P_{1}^{(n)}$.

Next, we turn to the second approximation function $P_{2}^{(n)}$, still in the case of $x>\ln K$. For the upper bound we use Lemma 35 as in the case $x \leq \ln K$ and proceed similarly to the proof of the upper bound for the first approximation function $P_{1}^{(n)}$. The proof of the lower bound is similar to the case $x \leq \ln K$ and we obtain the result observing that if $x>\ln K$ then $P(t, x)<\psi(x)+\delta=\delta$ for any $t \in[0, T]$.

\section{Computations}

In this section we exhibit computations of price functions and free boundaries of game and American put options. All graphs of functions related to game put options were plotted using the approximation function $P_{2}^{(2000)}$ (see (11)). The graphs for the American put options were computed using the approximation function $P_{A}^{(2000)}$ from [3].

Figure 1 shows both free boundaries of the holder and of the writer of a game put option and also the free boundary of the holder of an American put option corresponding to the option parameters $K=20, r=0.02, \kappa=0.15, T=$ 0.5 , and $\delta=0.15$. Here $K$ is the strike of the option, $r$ is the interest rate, $\kappa$ is the volatility, $T$ is the time to maturity, and $\delta$ is the writer's cancelation penalty in the case of game option. The horizontal axis represents here the time coordinate. The free boundary of the holder runs lower for American than for the game put option.

In Figure 2 we plot the graphs of an American put option price function and of a game put option price functions as functions of the initial value of the stock. Here $\delta=1.0$ and $\delta=1.5$, while other parameters are $K=20, r=0.02, \kappa=$ 0.15 , and $T=10$. To see what is what here we recall that prices of game options do not exceed prices of corresponding American options and higher penalties increase prices.

Figure 3 shows the holder's free boundary for American and game put options where we use the same setup and parameters as in Figure 1 adding also plots of free boundaries for the game put options with penalty values $\delta=0.3$ and $\delta=0.5$. To see which is which we observe that lower penalties lead to higher running holder's free boundaries in Figure 3, while the free boundary of the American put option remains the same here as in Figure 1.

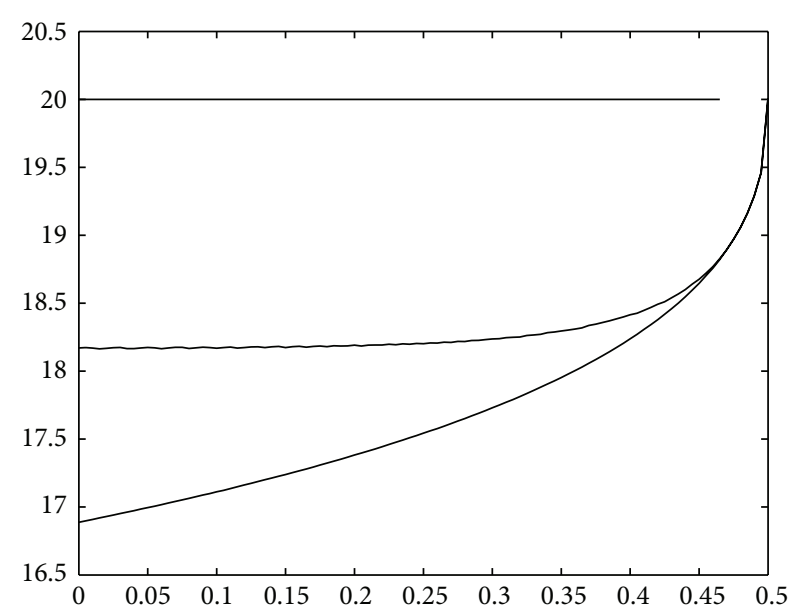

FIGURE 1: Free boundaries of American and game put options.

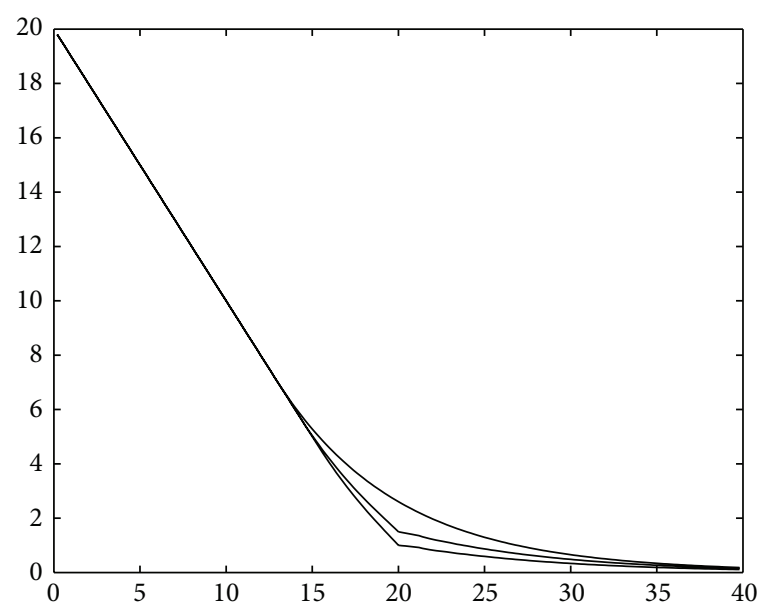

Figure 2: The price functions of American and game put options.

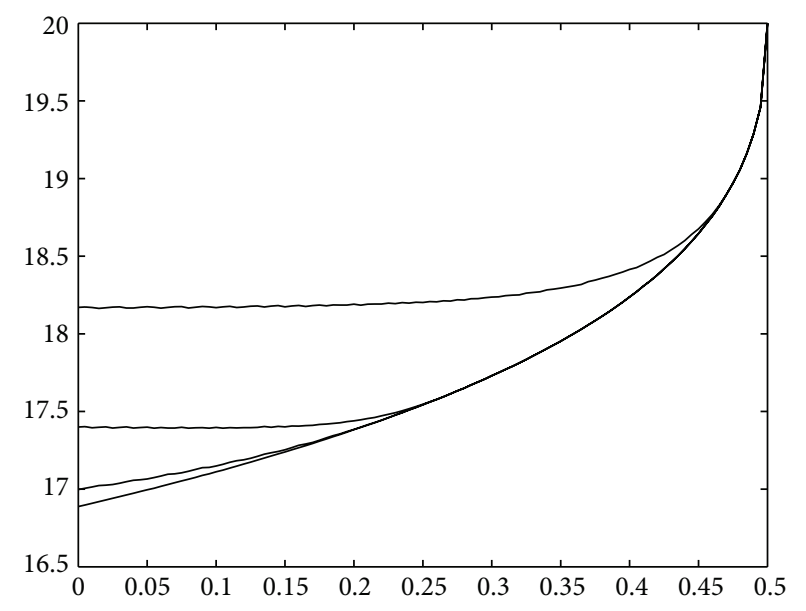

Figure 3: Holder free boundaries of American and game put options. 


\section{Conflict of Interests}

The authors declare that there is no conflict of interests regarding the publication of this paper.

\section{Acknowledgment}

This paper is partially supported by the ISF Grant no. 82/10.

\section{References}

[1] Y. Kifer, "Game options," Finance and Stochastics, vol. 4, no. 4, pp. 443-463, 2000.

[2] Y. Kifer, "Dynkin's games and Israeli options," ISRN Probability and Statistics, vol. 2013, Article ID 856458, 17 pages, 2013.

[3] D. Lamberton, "Error estimates for the binomial approximation of American put options," Annals of Applied Probability, vol. 8, no. 1, pp. 206-233, 1998.

[4] A. E. Kyprianou, "Some calculations for Israeli options," Finance and Stochastics, vol. 8, no. 1, pp. 73-86, 2004.

[5] Y. Kifer, "Error estimates for binomial approximations of game options," Annals of Applied Probability, vol. 16, no. 2, pp. 9841033, 2006.

[6] J.-F. Chassagneux, "A discreet-time approximation for doubly reflected BSDES," Advances in Applied Probability, vol. 41, no. 1, pp. 101-130, 2009.

[7] D. Kinderlehrer and G. Stampacchia, An Introduction to Variational Inequalities and Their Applications, vol. 88 of Pure and Applied Mathematics, Academic Press, New York, NY, USA, 1980.

[8] H. Kunita and S. Seko, "Game call options and their exercise regions," Tech. Rep. NANZAN-TR-2004-06, 2004.

[9] I. Karatzas and S. E. Shreve, Methods of Mathematical Finance, vol. 39 of Applications of Mathematics, Springer, New York, NY, USA, 1998.

[10] C. Kühn and A. E. Kyprianou, "Callable puts as composite exotic options," Mathematical Finance, vol. 17, no. 4, pp. 487-502, 2007.

[11] J. P. Lepeltier and J. P. Maingueneau, "Le jeu de Dynkin en theorie generale sans l'hypothese de Mokobodski," Stochastics, vol. 13, no. 1-2, pp. 24-44, 1984.

[12] A. N. Shiryayev, Probability, vol. 95 of Graduate Texts in Mathematics, Springer, New York, NY, USA, 1984.

[13] J. R. Cannon, The One-Dimensional Heat Equation, vol. 23 of Encyclopedia of Mathematics and its Applications, AddisonWesley, Reading, Mass, USA, 1984.

[14] A. Friedman, Partial Differential Equations of Parabolic Type, Prentice-Hall, Englewood Cliffs, NJ, USA, 1964.

[15] Y. Iron and Y. Kifer, "Hedging of swing game options in continuous time," Stochastics, vol. 83, no. 4-6, pp. 365-404, 2011.

[16] A. Bensoussan and A. Friedman, "Nonzero-sum stochastic differential games with stopping times and free boundary problems," Transactions of the American Mathematical Society, vol. 231, no. 2, pp. 275-327, 1977. 


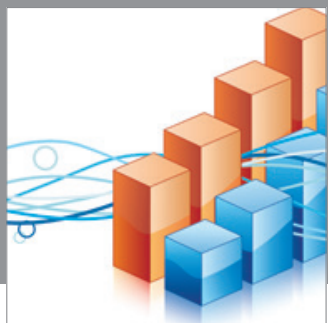

Advances in

Operations Research

mansans

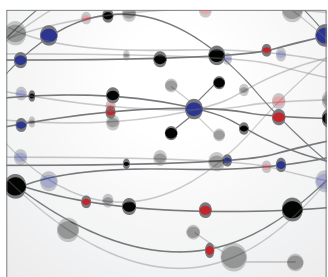

The Scientific World Journal
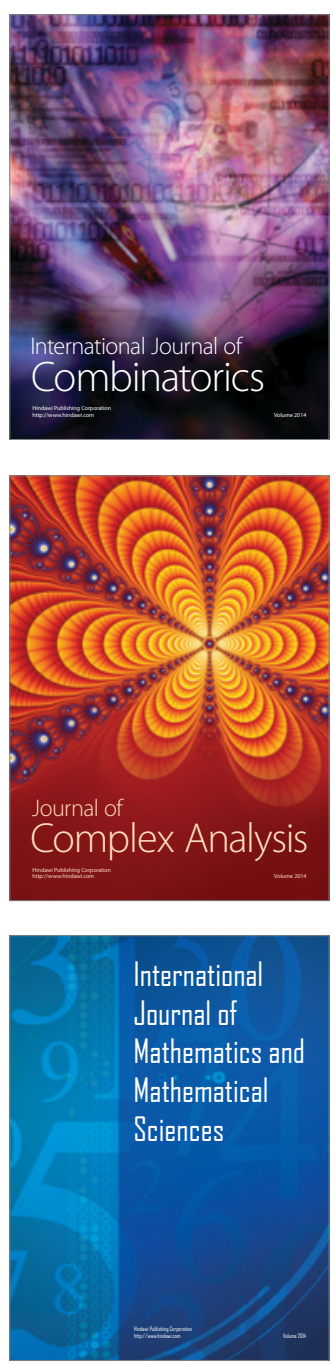
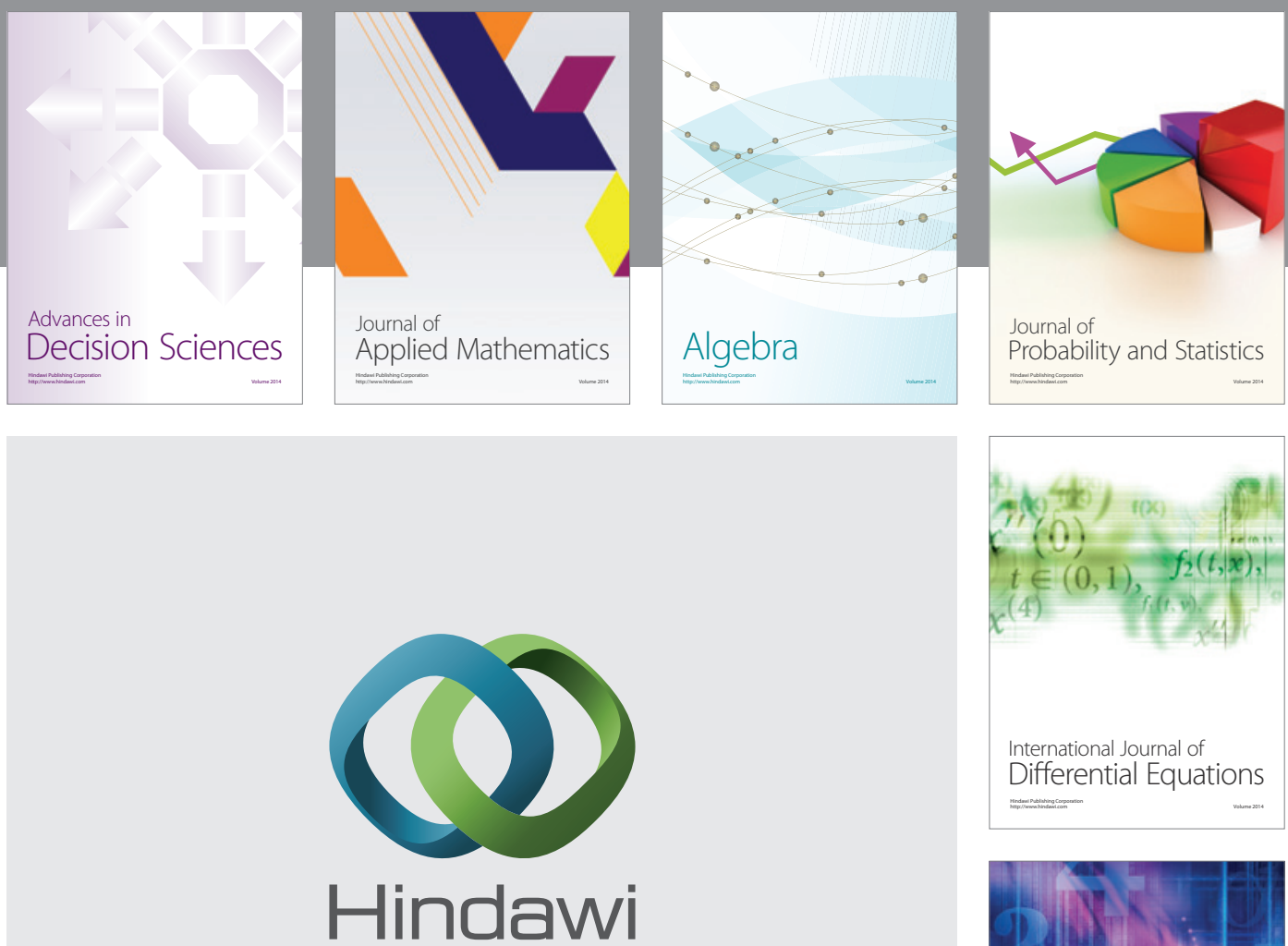

Submit your manuscripts at http://www.hindawi.com
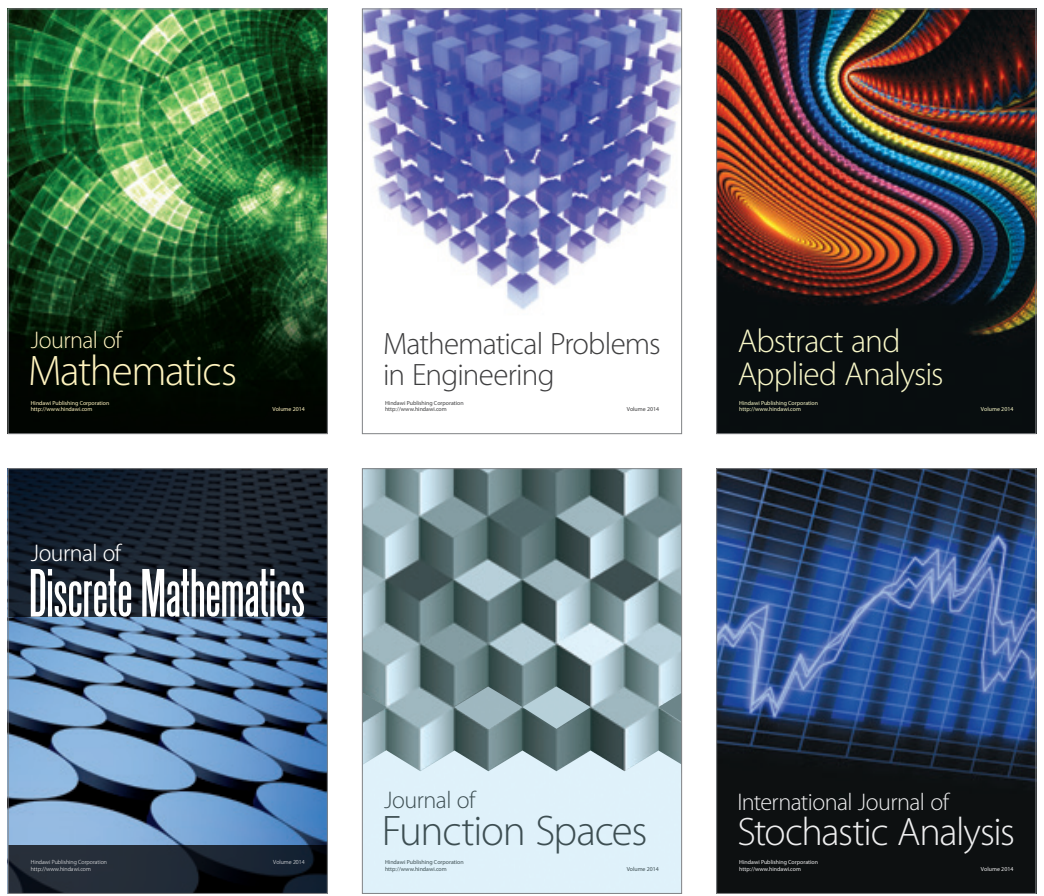

Journal of

Function Spaces

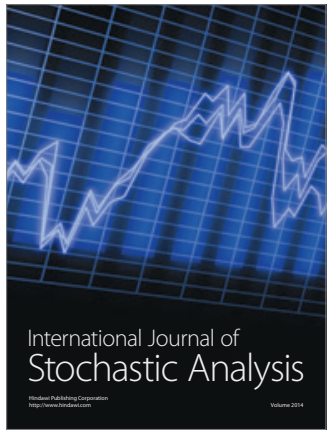

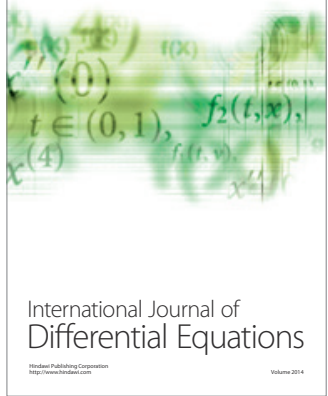
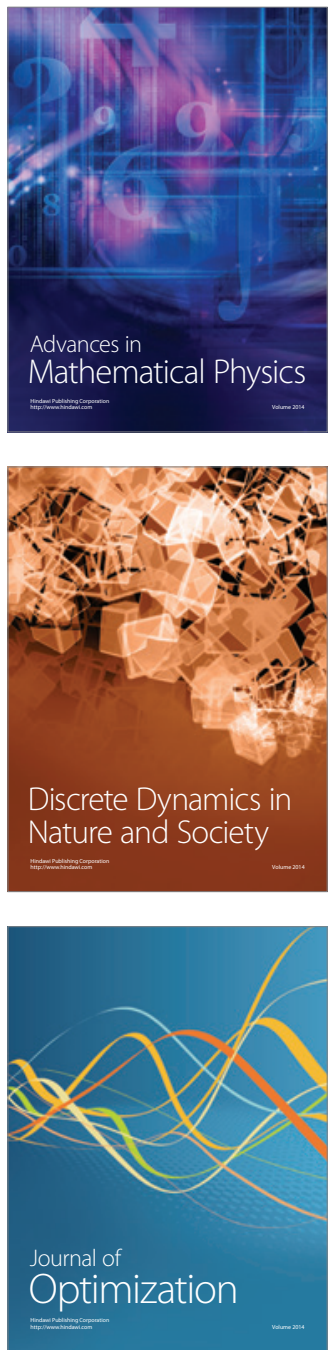